\title{
INDUCED TOPOLOGICAL PRESSURE FOR COUNTABLE STATE MARKOV SHIFTS
}

\author{
JOHANNES JAERISCH, MARC KESSEBÖHMER, AND SANAZ LAMEI
}

\begin{abstract}
We introduce the notion of induced topological pressure for countable state Markov shifts with respect to a non-negative scaling function and an arbitrary subset of finite words. Firstly, the scaling function allows a direct access to important thermodynamical quantities, which are usually given only implicitly by certain identities involving the classically defined pressure. In this context we generalise Savchenko's definition of entropy for special flows to a corresponding notion of topological pressure and show that this new notion coincides with the induced pressure for a large class of Hölder continuous height functions not necessarily bounded away from zero. Secondly, the dependence on the subset of words gives rise to interesting new results connecting the Gurevič and the classical pressure with exhausting principles for a large class of Markov shifts. In this context we consider dynamical group extentions to demonstrate that our new approach provides a useful tool to characterise amenability of the underlying group structure.
\end{abstract}

\section{INTRODUCTION AND STATEMENT OF MAIN RESULTS}

Throughout this paper we consider a topological Markov shift

$$
\Sigma:=\left\{\omega:=\left(\omega_{1}, \omega_{2}, \ldots\right) \in I^{\mathbb{N}}: \forall i \in \mathbb{N} A_{\omega_{i} \omega_{i+1}}=1\right\}
$$

with alphabet $I \subset \mathbb{N}$, incidence matrix $A \in\{0,1\}^{I \times I}$, and left shift map $\sigma: \Sigma \rightarrow \Sigma$ given by $\left(\omega_{1}, \omega_{2}, \ldots\right) \mapsto\left(\omega_{2}, \omega_{3}, \ldots\right)$. The shift map $\sigma$ is continuous with respect to the metric $d_{\alpha}\left(\omega_{1}, \omega_{2}\right):=\mathrm{e}^{-\alpha\left|\omega_{1} \wedge \omega_{2}\right|}, \alpha>0$, where $\omega_{1} \wedge \omega_{2}$ denotes the longest common initial block of $\omega_{1}, \omega_{2} \in \Sigma$. We denote the set of $A$-admissible words of length $n \in \mathbb{N}$ by $\Sigma^{n}:=\left\{\omega \in I^{n}: \forall i \in\{1, \ldots, n-1\}: A_{\omega_{i} \omega_{i+1}}=1\right\}$ and the set of $A$-admissible words of arbitrary length by $\Sigma^{*}:=\bigcup_{n \in \mathbb{N}} \Sigma^{n}$. For $\omega \in \Sigma^{*}$ we let $|\omega|$ denote the (word) length of $\omega$, which is the unique $n \in \mathbb{N}$ such that $\omega \in \Sigma^{n}$, and for $\omega \in \Sigma$ we set $|\omega|=\infty$. For $\omega \in \Sigma^{n}$ we call $[\omega]:=\left\{\tau \in \Sigma: \tau_{\mid n}=\omega\right\}$ the cylindrical set of $\omega$.

Let us now introduce the induced topological pressure, which is the central object of this paper.

2000 Mathematics Subject Classification. 37D35; 28A65.

Key words and phrases. thermodynamical formalism; topological pressure; countable state Markov shift; special flows; group extensions .

SL was supported by a grant offered by the University of Guilan. 
Definition 1.1. For $\varphi, \psi: \Sigma \rightarrow \mathbb{R}$ with $\psi \geq 0$, and $\mathcal{C} \subset \Sigma^{*}$ we define for $\eta>0$ the $\psi$-induced pressure of $\varphi$ (with respect to $\mathcal{C}$ ) by

$$
\mathcal{P}_{\psi}(\varphi, \mathcal{C}):=\limsup _{T \rightarrow \infty} \frac{1}{T} \log \sum_{\substack{\omega \in \mathcal{C} \\ T-\eta<S_{\omega} \psi \leq T}} \exp S_{\omega} \varphi,
$$

which takes values in $\overline{\mathbb{R}}:=\mathbb{R} \cup\{ \pm \infty\}$. In here, we set $S_{\omega} \varphi:=\sup _{\tau \in[\omega]} \sum_{k=0}^{|\omega|-1} \varphi \circ$ $\sigma^{k}(\tau)$.

It will follow from Theorem 2.4 that the definition of $\mathcal{P}_{\psi}(\varphi, \mathcal{C})$ is in fact independent of the choice of $\eta>0$. For this reason we are not going to refer to $\eta>0$ in the definition of the induced pressure.

Of particular interest for the choice of $\mathcal{C}$ is certainly the set of all finite words $\Sigma^{*}$ as well as the important subsets

$$
\Sigma^{\text {per }}:=\left\{\omega \in \Sigma^{*}: \bar{\omega} \in \Sigma\right\}, \quad \Sigma_{a}^{\text {per }}:=\left\{\omega \in \Sigma^{\text {per }}: \omega_{1}=a\right\} .
$$

In here, for $\omega=\left(\omega_{1}, \ldots, \omega_{n}\right) \in \Sigma^{*}$, we let $\bar{\omega}:=\left(\omega_{1}, \ldots, \omega_{n}, \omega_{1}, \ldots, \omega_{n}, \ldots\right)$ denote the periodic word in $I^{\mathbb{N}}$ with period $n \in \mathbb{N}$ and initial block $\omega$.

Our definition generalises known notions of topological pressure for the particular choice $\psi=1$. That is, if $A$ is mixing (i.e. $\forall i, j \in I \exists n_{0} \geq 1 \forall n \geq n_{0} \exists \omega \in \Sigma^{n}$ : $\left.i \omega j \in \Sigma^{*}\right)$ then $\mathcal{P}_{1}\left(\varphi, \Sigma_{a}^{\text {per }}\right)$ coincides with the Gurevič pressure (cf. [Sar99, Sar01]). Whereas $\mathcal{P}_{1}\left(\varphi, \Sigma^{*}\right)$ coincides with the classical notion of pressure for countable state Markov systems (cf. MU03|). It is important to note that in the context of the classical pressure one typically imposes that $A$ is finitely irreducible, i.e. there exists a finite set $\Lambda \subset \Sigma^{*}$ such that $\forall i, j \in I \exists \omega \in \Lambda: i \omega j \in \Sigma^{*}$. While in the context of the Gurevič pressure one typically imposes that $A$ is mixing and satisfies the big images and preimages (BIP) property, i.e. there exists a finite set $B \subset I$ such that $\forall i \in I \exists b_{1}, b_{2} \in B: b_{1} i b_{2} \in \Sigma^{*}$. Our general results make only few assumptions on the mixing properties of the Markov shift. We will show in Proposition 2.2 that several properties known for the 1-induced pressure allow a natural generalisation to the induced pressure.

Alternative approach to implicitly defined thermodynamic quantities

One important feature of the induced pressure is the freedom to choose a scaling function $\psi \geq 0$. This provides us with a new access to important thermodynamical quantities, which were so far only given implicitly as pseudo-inverse of the 1-induced pressure before. More precisely, under certain conditions (cf. Corollary 2.8, 2.10 and 2.12) we have

$$
\mathcal{P}_{\psi}(\varphi, \mathcal{C})=\inf \left\{\beta: \mathcal{P}_{1}(\varphi-\beta \psi, \mathcal{C}) \leq 0\right\} .
$$

In fact, the right-hand side is often referred to as the free energy, which is the main ingredient to determine multifractal spectra [JK11], large deviation asymptotics Kes01], or the thermodynamic formalism for special flows as elaborated in Remark 1.3 below. Furthermore, for the Gurevič case (i.e. $\mathcal{C}:=\Sigma_{a}^{\text {per }}$ ) we are able to provide in Proposition 3.26 a variational principle, which will turn out to be crucial for our study of special semi-flows.

Special semi-flows over countable state Markov shifts 
In this paragraph we will discuss the connection between the induced pressure and a new notion of pressure generalising Savchenko's definition of entropy. Let $\tau: \Sigma \rightarrow \mathbb{R}_{>0}$ be a continuous function and consider

$$
Y:=\left\{(\omega, t) \in \Sigma \times \mathbb{R}^{+}: 0 \leq t \leq \tau(\omega)\right\} / \sim,
$$

where we identify points via the equivalence relation given by $(w, \tau(w)) \sim(\sigma(w), 0)$. Let $\Phi=\left(\varphi_{t}\right)_{t \in \mathbb{R}_{>0}}$ be the special semi-flow over $Y$ with height function $\tau$ defined by $\varphi_{t}(\omega, s)=(\omega, s+t)$.

Savchenko was the first in Sav98] to define the topological entropy of special flows for non-compact spaces to be

$$
h(\Phi):=\sup \left\{h\left(\varphi_{1}, \mu\right): \mu \in \mathcal{E}_{\Phi}^{1}\right\},
$$

where $\mathcal{E}_{\Phi}^{1}$ denotes the set of $\Phi$-invariant ergodic probability measures on $Y$. Savchenko then proved in [Sav98] that, if $\tau$ depends only on the first coordinate and, then we have

$$
h(\Phi)=\inf \left\{\beta: \sum_{\omega \in \Sigma_{a}^{\mathrm{smpl}}} e^{-\beta S_{|\omega|} \tau} \leq 1\right\},
$$

where $\Sigma_{a}^{\mathrm{smpl}}:=\left\{\omega \in \Sigma_{a}^{\mathrm{per}}: \forall k \in \mathbb{N}: 1<k \leq|\omega|: \omega_{k} \neq a\right\}$.

This fact has been employed several times in the context of subgroups of the modular group; e.g. in [GK01], Iom10]. In [DL09a, DL09b] some techniques have been developed for computing the entropy of certain flows explicitly.

As a natural generalisation we may define the topological pressure of a continuous function $g: Y \rightarrow \mathbb{R}$ with respect to the special flow to be

$$
\mathbf{P}(g \mid \Phi):=\sup \left\{h\left(\varphi_{1}, \mu\right)+\int g d \mu: \mu \in \mathcal{E}_{\Phi}^{1} \text { such that } g \in L^{1}(\mu)\right\} .
$$

This gives in particular $h(\Phi)=\mathbf{P}(0 \mid \Phi)$.

With $\Delta_{g}: \Sigma \rightarrow \mathbb{R}$ given by $\Delta_{g}(\omega):=\int_{0}^{\tau(\omega)} g(\omega, t) d \lambda(t)$ we are in the position to state our main result concerning special flows.

Theorem 1.2. If $\tau: \Sigma \rightarrow \mathbb{R}_{>0}$ is Hölder continuous satisfying $\sum_{i=0}^{\infty} \tau \circ \sigma^{i}=\infty$ and $g: Y \rightarrow \mathbb{R}$ has the property that $\Delta_{g}: \Sigma \rightarrow \mathbb{R}$ is Hölder continuous then we have

$$
\mathbf{P}(g \mid \Phi)=\sup _{a \in I} \mathcal{P}_{\tau}\left(\Delta_{g}, \Sigma_{a}^{\text {per }}\right)=\sup _{\substack{Y \supset C \text { compact, } \\ \Phi-\text {-invariant }}} \mathbf{P}\left(\left.g\right|_{C}|\Phi|_{C}\right) .
$$

In particular, if $A$ is irreducible (i.e. $\forall i, j \in I \exists \omega \in \Sigma^{*}: i \omega j \in \Sigma^{*}$ ) then $\mathbf{P}(g \mid \Phi)=$ $\mathcal{P}_{\tau}\left(\Delta_{g}, \Sigma_{a}^{\text {per }}\right)$ for any $a \in I$.

The proof of Theorem 1.2 will be postponed to Section 4. It relies on some inducing techniques which allow us to overcome the difficulty of certain marginal measures on the base space to be infinite (see Lemma 4.1).

Remark 1.3. Using Corollary 3.19 we find that our definition of pressure for special flows in fact coincides with the definition in [BI06] of Barreira and Iommi, who considered a mixing base space $(\Sigma, \sigma)$, a height function $\tau$ bounded away from zero, and a function $g: Y \rightarrow \mathbb{R}$ such that $\Delta_{g}$ is Hölder continuous. That is

$$
\mathbf{P}(g \mid \Phi)=\inf \left\{\beta \in \mathbb{R}: \mathcal{P}_{1}\left(\Delta_{g}-\beta \tau, \Sigma_{a}^{\text {per }}\right) \leq 0\right\} .
$$


As a consequence of Theorem 1.2 and Corollary 3.19 we obtain that the above formula also holds true for the case where $A$ is only irreducible, and $\tau$ is not necessarily bounded away from zero and - different from Savchenko - may depend on more than one coordinate.

Exhausting principles, group extensions, and amenability

Another important feature of the induced pressure is the possibility to restrict appropriately to certain collections $\mathcal{C} \subset \Sigma^{*}$ of finite words. As we will discuss next, this provides us with a powerful tool to study for instance the structure of infinite group extensions.

Let us now focus on the relations for different choices of subcollections $\mathcal{C}, \mathcal{C}^{\prime} \subset \Sigma^{*}$. Recall from [HU99, MU01] that a sufficient condition for $\mathcal{P}_{1}\left(\varphi, \Sigma_{a}^{\text {per }}\right)$ and $\mathcal{P}_{1}\left(\varphi, \Sigma^{*}\right)$ to coincide is that the Markov shift is finitely irreducibe. As a general principle we will show in Corollary 3.23 that

$$
\mathcal{P}_{\psi}\left(\varphi, \Sigma^{*}\right)=\mathcal{P}_{\psi}\left(\varphi, \Sigma_{a}^{\text {per }}\right) \Longleftrightarrow\left(\psi, \varphi, \Sigma^{*}\right) \text { satisfies the exhausting principle. }
$$

We say that the exhausting principle holds for $(\psi, \varphi, \mathcal{C})$, if there exists a sequence $\left(K_{n}\right)_{n \in \mathbb{N}}$ of compact $\sigma$-invariant subsets of $\Sigma$, such that

$$
\lim _{n \rightarrow \infty} \mathcal{P}_{\psi, K_{n}}(\varphi, \mathcal{C})=\mathcal{P}_{\psi}(\varphi, \mathcal{C}), \text { where } \mathcal{P}_{\psi, K}(\varphi, \mathcal{C}):=\mathcal{P}_{\left.\psi\right|_{K}}\left(\left.\varphi\right|_{K}, \mathcal{C} \cap K^{*}\right),
$$

with $K^{*}:=\left\{\omega \in \Sigma^{*}:[\omega] \cap K \neq \emptyset\right\}$. A sufficient condition for the exhausting principle to hold for $\left(\psi, \varphi, \Sigma^{*}\right)$ and arbitrary Hölder continuous functions $\psi>0$ and $\varphi$ is that $A$ is finitely irreducible (see Corollary 3.13) and hence this fact gives a generalisation of the observation in [MU03, Theorem 2.1.5] to our context. However, Example 3.25 will show that this condition is not necessary.

In fact, the above observation is only a special case of a more general setting. For this we consider collections $\mathcal{C}^{\prime} \subset \mathcal{C} \subset \Sigma^{*}$, which are representable by so-called $\mathcal{C}$ loops (see Definition 3.1). See Theorem 3.12 for the corresponding main results. One of our purposes to introduce this general framework is to study skew product dynamical systems connected to infinite group extensions. More precisely, we consider certain shift spaces involving the structure of a finitely generated group $G:=\mathbb{F}_{k} / N$, where $\mathbb{F}_{k}$ denotes the free group with $k \in \mathbb{N}$ generators. For a natural choice of $\mathcal{C}^{\prime} \subset \mathcal{C}$ of finite words we obtain in Theorem 3.17 that

$$
\begin{aligned}
G \text { is amenable } & \Longleftrightarrow(1,0, \mathcal{C}) \text { satisfies the exhausting principle } \\
& \Longleftrightarrow \mathcal{P}_{1}(0, \mathcal{C})=\mathcal{P}_{1}\left(0, \mathcal{C}^{\prime}\right) .
\end{aligned}
$$

Moreover, we have that $\mathcal{C}$ is finitely irreducible (see Definition 3.5), if and only if the group $G$ is finite. We would like to point out that Theorem 3.17 provides us with a large class of interesting examples for which the exhausting principle holds, while the underlying Markov shift is far from being finitely irreducible. In Example 3.16 we elaborate this idea explicitly.

\section{BASIC PROPERTIES OF THE INDUCED PRESSURE}

In this section we will investigate the basic properties of the $\psi$-induced pressure for non-negative scaling functions $\psi: \Sigma \rightarrow \mathbb{R}$ and subsets $\mathcal{C} \subset \Sigma^{*}$. 
We call a function $f: \Sigma \rightarrow \mathbb{R} \alpha$-Hölder continuous, if there exists $\alpha>0$ and a constant $V_{\alpha}(f)$, such that for all $\omega, \omega^{\prime} \in \Sigma$

$$
\left|f(\omega)-f\left(\omega^{\prime}\right)\right| \leq e^{-\alpha} V_{\alpha}(f) d_{\alpha}\left(\omega, \omega^{\prime}\right) .
$$

We call $f$ Hölder continuous, if there exists $\alpha>0$ such that $f$ is $\alpha$-Hölder continuous.

The bounded distortion lemma (see e.g. [MU03, Lemma 2.3.1]) shows that for a Hölder continuous function $f$ we have for every $\omega \in \Sigma^{*}$ and $\tau, \tau^{\prime} \in[\omega]$

$$
\left|S_{|\omega|} f(\tau)-S_{|\omega|} f\left(\tau^{\prime}\right)\right| \leq \frac{V_{\alpha}(f)}{e^{\alpha}-1} d_{\alpha}\left(\sigma^{|\omega|}(\tau), \sigma^{|\omega|}\left(\tau^{\prime}\right)\right) .
$$

This in particular implies the existence of a constant $C_{f}>0$, such that

$$
\left|S_{|\omega|} f(\tau)-S_{|\omega|} f\left(\tau^{\prime}\right)\right| \leq C_{f}
$$

for all $\omega \in \Sigma^{*}$ and $\tau, \tau^{\prime} \in[\omega]$. We will refer to (2.3) as the bounded distortion property.

Remark 2.1. If $\varphi$ and $\psi$ are both Hölder continuous and $\mathcal{C} \subset \Sigma^{*}$, then in the definition of $\mathcal{P}_{\psi}(\varphi, \mathcal{C})$ we may replace $S_{\omega} \varphi$ by $\inf _{\tau \in[\omega]} \sum_{k=0}^{|\omega|-1} \varphi \circ \sigma^{k}(\tau)$ without altering the value of the pressure. If, additionally, $\mathcal{C}:=\Sigma^{\text {per }}$ or $\Sigma_{a}^{\text {per }}, a \in I$, then we may also use $\sum_{k=0}^{|\omega|-1} \varphi \circ \sigma^{k}(\bar{\omega})$ for $\omega \in \Sigma^{\text {per }}$. Independent of the particular choice for $S_{\omega} \varphi$ we may also choose $S_{\omega} \psi$ in an analogue fashion.

Also notice, that, if $\varphi$ and $\psi$ are both Hölder continuous, then we have for the same reason $\mathcal{P}_{\psi, K}(\varphi, \mathcal{C})=\mathcal{P}_{\psi}\left(\varphi, \mathcal{C} \cap K^{*}\right)$ for all $\sigma$-invariant subsets $K \subset \Sigma$.

The following properties of the induced pressure are similar to the well-known properties of the classical topological pressure (cf. Wal82, Sec. 9.2]).

Proposition 2.2. Let $\varphi, \varphi_{1}, \varphi_{2}, \psi, \psi_{1}, \psi_{2}$ be real functions on $\Sigma$. The induced topological pressure has the following basic properties.

(1) (Monotonicity) For $\varphi_{1} \leq \varphi_{2}, \psi_{2} \geq \psi_{1} \geq 0, \mathcal{C}_{1} \subset \mathcal{C}_{2} \subset \Sigma^{*}$, and $K_{1} \subset K_{2} \subset$ $\Sigma \sigma$-invariant, we have,

$$
\mathcal{P}_{\psi_{1}, K_{1}}\left(\varphi_{1}, \mathcal{C}_{1}\right) \leq \mathcal{P}_{\psi_{2}, K_{2}}\left(\varphi_{2}, \mathcal{C}_{2}\right)
$$

(2) (Continuity) If $\psi \geq c>0$, or $\psi>0$ and $\varphi$ are both Hölder continuous and $\mathcal{C}=\Sigma^{*}$, then

$$
\mathcal{P}_{(\cdot)}(\cdot, \mathcal{C}): C(\Sigma, \mathbb{R}) \times C(\Sigma, \mathbb{R}) \rightarrow \overline{\mathbb{R}}
$$

is continuous in $(\varphi, \psi)$ with respect to the product topology, where the set of continuous functions $C(\Sigma, \mathbb{R})$ is equipped with the $\|\cdot\|_{\infty}-$ norm.

(3) (Convexity) For $\psi \geq 0$ and $\varphi_{1}, \varphi_{2} \in C(\Sigma, \mathbb{R})$ with $\mathcal{P}_{\psi}\left(\varphi_{i}, \mathcal{C}\right)>-\infty$, for $i \in\{1,2\}$ and $t \in(0,1)$ we have

$$
\mathcal{P}_{\psi}\left(t \varphi_{1}+(1-t) \varphi_{2}, \mathcal{C}\right) \leq t \mathcal{P}_{\psi}\left(\varphi_{1}, \mathcal{C}\right)+(1-t) \mathcal{P}_{\psi}\left(\varphi_{2}, \mathcal{C} .\right)
$$

(4) (Translation) If $0<m \leq \psi \leq M$ then

$$
\mathcal{P}_{\psi}(\varphi, \mathcal{C})+c / M \leq \mathcal{P}_{\psi}(\varphi+c, \mathcal{C}) \leq \mathcal{P}_{\psi}(\varphi, \mathcal{C})+c / m .
$$

(5) (Subadditivity) We have

$$
\mathcal{P}_{\psi}\left(\varphi_{1}+\varphi_{2}, \mathcal{C}\right) \leq \mathcal{P}_{\psi}\left(\varphi_{1}, \mathcal{C}\right)+\mathcal{P}_{\psi}\left(\varphi_{2}, \mathcal{C}\right)
$$


(6) (Stability) For $\mathcal{C}_{i} \subset \Sigma^{*}, i \in\{1, \ldots, n\}$, we have

$$
\mathcal{P}_{\psi}\left(\varphi, \bigcup_{i=1}^{n} \mathcal{C}_{i}\right)=\max \left\{\mathcal{P}_{\psi}\left(\varphi, \mathcal{C}_{i}\right): i \in\{1, \ldots, n\}\right\}
$$

(7) (Subhomogeneity) If $\mathcal{P}_{\psi}(\varphi, \mathcal{C}) \in \mathbb{R}$ then $\mathcal{P}_{\psi}(c \varphi, \mathcal{C}) \leq c \mathcal{P}_{\psi}(\varphi, \mathcal{C})$, for $c \geq 1$, and $\mathcal{P}_{\psi}(c \varphi, \mathcal{C}) \geq c \mathcal{P}_{\psi}(\varphi, \mathcal{C})$, for $c \leq 1$

(8) (Bounded cocycle) Let us write $\varphi_{1} \sim \varphi_{2}$ for $\varphi_{1}, \varphi_{2} \in C(\Sigma, \mathbb{R})$, if $\varphi_{1}$ and $\varphi_{2}$ are cohomologous in the class of bounded continuous functions, i.e. there exists a bounded function $h \in C(\Sigma, \mathbb{R})$ such that $\varphi_{1}=\varphi_{2}+h-h \circ \sigma$. For $\varphi_{1} \sim \varphi_{2}$, we have

$$
\mathcal{P}_{\psi}\left(\varphi_{1}, \mathcal{C}\right)=\mathcal{P}_{\psi}\left(\varphi_{2}, \mathcal{C}\right)
$$

if $\varphi \sim \psi$ then we have, for every $t \in \mathbb{R}$,

$$
\mathcal{P}_{\psi}(t \varphi, \mathcal{C})=t+\mathcal{P}_{\psi}(0, \mathcal{C})
$$

Proof. The proofs of (4), (5) and (7) are straightforward generalisations of the corresponding statements for the classical pressure (cf. [Wal82, Sec. 9.2]). (11), (6) and (8) follow immediately from the definition of the $\psi$-induced pressure. Hence, let us only address (2) and (3).

ad (2): By Corollary 2.10 below we have

$$
\mathcal{P}_{\psi}(\varphi, \mathcal{C})=\inf \left\{\beta: \mathcal{P}_{1}(\varphi-\beta \psi, \mathcal{C}) \leq 0\right\}=\sup \left\{\beta: \mathcal{P}_{1}(\varphi-\beta \psi, \mathcal{C}) \geq 0\right\} .
$$

The assertion follows, since $\mathcal{P}_{1}(\cdot, \mathcal{C}): C(\Sigma, \mathbb{R}) \rightarrow \overline{\mathbb{R}}$ is continuous with respect to the $\|\cdot\|_{\infty}$-norm.

ad (3): The assumption $\mathcal{P}_{\psi}\left(\varphi_{i}, \mathcal{C}\right)>-\infty$ for $i \in\{1,2\}$ implies that there exists a sequence $\left(T_{k}\right)$ tending to infinity such that the sets $\left\{\omega \in \mathcal{C}: T_{k}-\eta<S_{\omega} \psi \leq T_{k}\right\}$ are non empty and

$$
\lim _{k \rightarrow \infty} \frac{1}{T_{k}} \log \sum_{\substack{\omega \in \mathcal{C} \\ T_{k}-\eta<S_{\omega} \leq T_{k}}} \exp S_{\omega}\left(t \varphi_{1}+(1-t) \varphi_{2}\right)=\mathcal{P}_{\psi}\left(t \varphi_{1}+(1-t) \varphi_{2}, \mathcal{C}\right) .
$$

By Hölder's inequality we have

$$
\begin{aligned}
\sum_{\substack{\omega \in \mathcal{C} \\
T_{k}-\eta<S_{\omega} \psi T_{k}}} \mathrm{e}^{S_{\omega}\left(t \varphi_{1}+(1-t) \varphi_{2}\right)} & \leq \sum_{\substack{\omega \in \mathcal{C} \\
T_{k}-\eta<S_{\omega} \psi \leq T_{k}}} \mathrm{e}^{S_{\omega} t \varphi_{1}} \mathrm{e}^{S_{\omega}(1-t) \varphi_{2}} \\
& \leq\left(\sum_{\substack{\omega \in \mathcal{C} \\
T_{k}-\eta<S_{\omega} \leq T_{k}}} \mathrm{e}^{S_{\omega} \varphi_{1}}\right)^{t}\left(\sum_{\substack{\left.\omega \in \mathcal{C} \\
T_{k}-\eta<S_{\omega}\right\} \leq T_{k}}} \mathrm{e}^{S_{\omega} \varphi_{2}}\right)^{1-t} .
\end{aligned}
$$

Taking logarithm, dividing by $T_{k}$ completes the proof.

Remark 2.3. For the multifractal analysis in the setting of conformal iterated function systems the free energy function studied in [JK11] coincides with $\beta \mapsto$ $\mathcal{P}_{-\zeta}\left(\beta \psi, \Sigma^{*}\right)$ by Corollary 2.8 below, where $\zeta$ denotes the geometric potential associated to the conformal iterated function system and $\psi$ is the potential defining the level sets under consideration. The Legendre transform of the free energy function describes the dimension spectrum of the multifractal level sets. Proposition 2.2 (3) in particular implies that the free energy function is convex, see also [JK11, Lemma 3.1 . 
The main result in this section is that the $\psi$-induced pressure coincides with a certain critical exponent of the partition function as stated in the following theorem. We remark that [MU03, Theorem 2.1.3] is covered by our theorem by choosing $\psi=1$ (see Remark 2.7 for further comments). We would like to remark that a similar connection has been considered by Przytycki in [Prz99] to introduce the notion of Poincaré exponent for rational functions.

Theorem 2.4 (Critical exponent). For potential functions $\varphi, \psi: \Sigma \rightarrow \mathbb{R}$ satisfying $\psi \geq 0$ we have

$$
\mathcal{P}_{\psi}(\varphi, \mathcal{C})=\inf \left\{\beta \in \mathbb{R}: \limsup _{T \rightarrow \infty} \sum_{\substack{\omega \in \mathcal{C} \\ T<S_{\omega} \psi}} \exp \left(S_{\omega} \varphi-\beta S_{\omega} \psi\right)<\infty\right\} .
$$

In particular, the definition of $\mathcal{P}_{\psi}(\varphi, \mathcal{C})$ is independent of the choice of $\eta>0$.

Proof. Fix $\eta>0$. For $\omega \in \mathcal{C}$ let $n(\omega)$ denote the unique $n \in \mathbb{N}$, such that $(n-1) \eta<$ $S_{\omega} \psi(\omega) \leq n \eta$. Observing that $\mathrm{e}^{-\beta n(\omega) \eta} \mathrm{e}^{-|\beta| \eta} \leq \mathrm{e}^{-\beta S_{\omega} \psi} \leq \mathrm{e}^{-\beta n(\omega) \eta} \mathrm{e}^{|\beta| \eta}$ for all $\omega \in \mathcal{C}$ we conclude that

$$
\limsup _{T \rightarrow \infty} \sum_{\substack{\omega \in \mathcal{C} \\ T<S_{\omega} \psi}} \exp \left(S_{\omega} \varphi-\beta S_{\omega} \psi\right)<\infty
$$

if and only if

$$
\limsup _{N \rightarrow \infty} \sum_{n \geq N} \sum_{\substack{\omega \in \mathcal{C} \\(n-1) \eta<S \omega \psi \leq n \eta}} \exp \left(S_{\omega} \varphi-\beta n \eta\right)<\infty .
$$

Hence, it will be sufficient to verify that

$$
\mathcal{P}_{\psi}(\varphi, \mathcal{C})=\inf \left\{\beta \in \mathbb{R}: \limsup _{N \rightarrow \infty} \sum_{n \geq N} \sum_{\substack{\omega \in \mathcal{C} \\(n-1) \eta<S_{\omega} \psi \leq n \eta}} \exp \left(S_{\omega} \varphi-\beta n \eta\right)<\infty\right\}
$$

For the " $\leq$ " part we will show that for every $\beta \in \mathbb{R}$ and $\epsilon>0$ such that $\beta<$ $\mathcal{P}_{\psi}(\varphi, \mathcal{C})-\epsilon$, we have for all $N \in \mathbb{N}$,

$$
\sum_{n \geq N} \sum_{\substack{\omega \in \mathcal{C} \\(n-1) \\ \eta<S_{\omega} \psi \leq n \eta}} \exp \left(S_{\omega} \varphi-\beta n \eta\right)=\infty
$$

By the definition of $\mathcal{P}_{\psi}(\varphi, \mathcal{C})$ there exists a sequence $\left(T_{j}\right)_{j \in \mathbb{N}}$, such that for every $j \in \mathbb{N}, T_{j+1}-T_{j}>\eta$ and

$$
\frac{1}{T_{j}} \log \sum_{\substack{\omega \in \mathcal{C} \\ T_{j}-\eta<S_{\omega \psi} \leq T_{j}}} \exp \left(S_{\omega} \varphi\right) \geq \beta+\epsilon .
$$


For $\omega \in \mathcal{C}$ with $T_{j}-\eta<S_{\omega} \psi \leq T_{j}$ we have $\left|n(\omega) \eta-T_{j}\right|<2 \eta$ and hence

$$
\begin{aligned}
& \sum_{n \geq N_{(n-1) \eta<S_{\omega} \psi(\omega) \leq n \eta}} \sum_{\substack{\omega \in \mathcal{C} \\
S^{S_{\omega} \varphi-\beta n \eta}}} \geq \sum_{j \in \mathbb{N}: T_{j} \geq N \eta} \sum_{\substack{\omega \in \mathcal{C} \\
T_{j}-\eta<S_{\omega} \psi \leq T_{j}}} \exp \left(S_{\omega} \varphi-\beta n(\omega) \eta\right), \\
& \\
& \geq \mathrm{e}^{-2 \eta|\beta|} \sum_{j \in \mathbb{N}: T_{j} \geq N \eta} \sum_{\substack{\omega \in \mathcal{C} \\
T_{j}-\eta<S_{\omega} \psi T_{j}}} \exp \left(S_{\omega} \varphi-\beta T_{j}\right) \\
&=\infty .
\end{aligned}
$$

This argument is not only valid for $\mathcal{P}_{\psi}(\varphi, \mathcal{C}) \in \mathbb{R}$, but also for $\mathcal{P}_{\psi}(\varphi, \mathcal{C})=\infty$, in which case (2.4) holds for every $\beta \in \mathbb{R}$.

For the " $\geq$ " part we first consider the case $\mathcal{P}_{\psi}(\varphi, \mathcal{C})>-\infty$ and show that for every $\epsilon>0$

$$
\limsup _{N \rightarrow \infty} \sum_{n \geq N} \sum_{\substack{\omega \in \mathcal{C} \\(n-1) \eta<S_{\omega} \psi \leq n \eta}} \exp \left(S_{\omega} \varphi-\left(\mathcal{P}_{\psi}(\varphi, \mathcal{C})+\epsilon\right) n \eta\right)<\infty .
$$

Again, by the definition of $\mathcal{P}_{\psi}(\varphi, \mathcal{C})$ we find for all $\epsilon>0$ an element $N \in \mathbb{N}$, such that for all $n \geq N$

$$
\sum_{\substack{\omega \in \mathcal{C} \\(n-1) \eta<S_{\omega} \leqslant \leq n \eta}} \exp S_{\omega} \varphi \leq \exp \left(n \eta\left(\mathcal{P}_{\psi}(\varphi, \mathcal{C})+\epsilon / 2\right)\right) .
$$

Consequently, we have

$$
\begin{aligned}
\sum_{n \geq N} & \sum_{\substack{\omega \in \mathcal{C} \\
(n-1) \eta<S_{\omega} \psi \leq n \eta}} \exp \left(S_{\omega} \varphi-\left(\mathcal{P}_{\psi}(\varphi, \mathcal{C})+\epsilon\right) n \eta\right) \\
& \leq \sum_{n \geq N} \exp \left(-n \eta\left(\mathcal{P}_{\psi}(\varphi, \mathcal{C})+\epsilon\right)\right) \exp \left(n \eta\left(\mathcal{P}_{\psi}(\varphi, \mathcal{C})+\epsilon / 2\right)\right) \\
& =\sum_{n \geq N} \exp (-n \eta \epsilon / 2)<\infty .
\end{aligned}
$$

For the case $\mathcal{P}_{\psi}(\varphi, \mathcal{C})=-\infty$ we have for all $\rho \in \mathbb{R}$ and all $n \in \mathbb{N}$ sufficiently large that $\sum_{\substack{\left.\omega \in \mathcal{C} \\(n-1) \eta<S_{\omega}\right\} \leq n \eta}} \exp S_{\omega} \varphi \leq \exp (n \eta \rho)$. Hence, by the same arguments we conclude that for all $\beta \in \mathbb{R}$ and $N$ sufficiently large we have convergence of the series

$$
\sum_{n \geq N} \sum_{\substack{\left.\omega \in \mathcal{C} \\(n-1) \eta<S_{\omega}\right\} \leq n \eta}} \exp \left(S_{\omega} \varphi-\beta n \eta\right)
$$

Remark 2.5. The previous proof in particular shows that it is sufficient to take the limes superior with respect to the subsequence $T_{k}=\eta k, k \in \mathbb{N}, \eta>0$. More precisely, for potential functions $\varphi, \psi: \Sigma \rightarrow \mathbb{R}$ with $\psi \geq 0$ and for any $\eta>0$ we have

$$
\mathcal{P}_{\psi}(\varphi, \mathcal{C})=\limsup _{n} \frac{1}{n \eta} \log \sum_{\substack{\omega \in \mathcal{C} \\(n-1) \eta<S_{\omega} \psi \leq n \eta}} \exp S_{\omega} \varphi .
$$

The next Lemma gives an alternative way to describe the $\psi$-induced pressure, which will prove to be useful subsequently. 
Lemma 2.6. Let $\varphi, \psi: \Sigma \rightarrow \mathbb{R}$ be Hölder continuous and $\psi \geq 0$ and $\mathcal{C} \subset \Sigma^{*}$. If $\mathcal{P}_{\psi}(\varphi, \mathcal{C}) \geq 0$ then for all sufficiently large $T>0$ we have

$$
\mathcal{P}_{\psi}(\varphi, \mathcal{C})=\limsup _{n \rightarrow \infty} \frac{1}{n} \log \sum_{\substack{\omega \in \mathcal{C} \\ T<S_{\omega} \psi \leq n}} \exp S_{\omega} \varphi
$$

and for $\mathcal{P}_{\psi}(\varphi, \mathcal{C})<0$ we have

$$
\mathcal{P}_{\psi}(\varphi, \mathcal{C})=\limsup _{n \rightarrow \infty} \frac{1}{n} \log \sum_{\substack{\omega \in \mathcal{C} \\ n \leq S_{\omega} \psi}} \exp S_{\omega} \varphi
$$

Proof. By the definition of $\mathcal{P}_{\psi}(\varphi, \mathcal{C})$ we find for $\epsilon>0$ an element $N \in \mathbb{N}$, such that for all $n \geq N$

$$
\sum_{\substack{\omega \in \mathcal{C} \\ n-1<S_{\omega} \psi \leq n}} \exp S_{\omega} \varphi \leq \exp \left(n\left(\mathcal{P}_{\psi}(\varphi, \mathcal{C})+\epsilon\right)\right) .
$$

Let us begin with the case $\mathcal{P}_{\psi}(\varphi, \mathcal{C}) \geq 0$. Then (2.6) implies

$$
\sum_{\substack{\omega \in \mathcal{C} \\ N-1<S_{\omega} \psi \leq n}} \mathrm{e}^{S_{\omega} \varphi} \leq \sum_{k=N-1}^{n} \mathrm{e}^{k\left(\mathcal{P}_{\psi}(\varphi, \mathcal{C})+\epsilon\right)} \leq \frac{\exp \left((n+1)\left(\mathcal{P}_{\psi}(\varphi, \mathcal{C})+\epsilon\right)\right)-1}{\exp \left(\mathcal{P}_{\psi}(\varphi, \mathcal{C})+\epsilon\right)-1}
$$

Since $\mathcal{P}_{\psi}(\varphi, \mathcal{C})+\epsilon>0$ we may take logarithm and divide by $n$ to obtain the upper bound.

For the case $\mathcal{P}_{\psi}(\varphi, \mathcal{C})<0$ fix $\epsilon \in\left(0,-\mathcal{P}_{\psi}(\varphi, \mathcal{C})\right)$. This time (2.6) implies for $n \geq N$

$$
\sum_{\substack{\omega \in \mathcal{C} \\ n \leq S_{\omega} \psi}} \mathrm{e}^{S_{\omega} \varphi} \leq \sum_{k=n}^{\infty} \mathrm{e}^{k\left(\mathcal{P}_{\psi}(\varphi, \mathcal{C})+\epsilon\right)}=\frac{\exp \left(n\left(\mathcal{P}_{\psi}(\varphi, \mathcal{C})+\epsilon\right)\right)}{1-\exp \left(\mathcal{P}_{\psi}(\varphi, \mathcal{C})+\epsilon\right)}
$$

and hence taking logarithm and dividing by $n$ finishes the proof for the upper bound. The lower bound is immediate in both cases.

Remark 2.7. We would like to compare Theorem 2.4 with [MU03, Theorem 2.1.3] and also the classical definition of the Poincaré exponent. Under the condition that $\lim \sup _{n} \sum_{\substack{\omega \in \mathcal{C} \\ n-1<S_{\omega} \leq n}} \mathrm{e}^{S_{\omega} \varphi}<\infty$ implies that $\sum_{\substack{\left.\omega \in \mathcal{C} \\ n-1<S_{\omega}\right\} \leq n}} \mathrm{e}^{S_{\omega} \varphi}<\infty$ for all $n \in \mathbb{N}$ we have by Theorem 2.4

$$
\mathcal{P}_{\psi}\left(\varphi, \Sigma^{*}\right)=\inf \left\{\beta \in \mathbb{R}: \sum_{\omega \in \Sigma^{*}} \exp \left(S_{\omega} \varphi-\beta S_{\omega} \psi\right)<\infty\right\}
$$

and by Lemma 2.6, for $\mathcal{P}_{\psi}(\varphi, \mathcal{C}) \geq 0$,

$$
\mathcal{P}_{\psi}(\varphi, \mathcal{C})=\limsup _{n \rightarrow \infty} \frac{1}{n} \log \sum_{\substack{\omega \in \mathcal{C} \\ S \omega \psi \leq n}} \exp S_{\omega} \varphi .
$$

Generalising the proof of [MU03, Proposition 2.1.9] this is in particular the case for $A$ finitely irreducible, $\mathcal{C}=\Sigma^{*}$, and $\psi \geq c>0$. In general, the identity (2.7) does not hold. For instance, consider the renewal shift with state space $\mathbb{N}$ rooted at 1 , which is mixing but not finitely irreducible. Next choose the potential functions $\varphi_{[k]}:=-\log k$ for $k \geq 2$ and $\varphi_{\mid[1, n]}:=-2 \log n$ for $n \geq 1$. Then $\mathcal{P}_{1}\left(\varphi, \Sigma^{*}\right) \leq \frac{1}{2} \log \sum_{|\omega|=2} \mathrm{e}^{S_{\omega} \varphi}=\frac{1}{2} \log \left(\sum_{k \geq 2} \mathrm{e}^{S_{(k, k-1)} \varphi}+\sum_{n \geq 1} \mathrm{e}^{S_{(1, n)} \varphi}\right)=$ 
$\frac{1}{2} \log \left(\sum_{k \geq 2} 1 / k(k-1)+\sum_{n \geq 1} 1 / n^{2}\right)<\infty$, whereas for all $\beta \in \mathbb{R}$ we have $\sum_{\omega \in \Sigma^{*}} \mathrm{e}^{S_{\omega} \varphi-\beta|\omega|} \geq$ $\sum_{k \geq 2} 1 / k=\infty$ implying that in this situation the right hand side of (2.7) is equal to infinity.

Corollary 2.8. For potential functions $\varphi, \psi: \Sigma \rightarrow \mathbb{R}, \psi \geq 0$, we always have

$$
\mathcal{P}_{\psi}(\varphi, \mathcal{C}) \geq \inf \left\{\beta \in \mathbb{R}: \mathcal{P}_{1}(\varphi-\beta \psi, \mathcal{C}) \leq 0\right\}
$$

Proof. Since

$$
\begin{aligned}
\inf \left\{\beta \in \mathbb{R}: \limsup _{T \rightarrow \infty} \sum_{\substack{\omega \in \mathcal{C} \\
T<S_{\omega} \psi}} \exp \left(S_{\omega} \varphi-\beta S_{\omega} \psi\right)\right. & <\infty\} \\
& \geq \inf \left\{\beta \in \mathbb{R}: \mathcal{P}_{1}(\varphi-\beta \psi, \mathcal{C}) \leq 0\right\}
\end{aligned}
$$

the inequality (2.8) follows by Theorem 2.4 .

We say that $\mathcal{P}_{\psi}(\varphi, \mathcal{C})$ is given by the pseudo inverse (of the 1 -induced pressure), if $\mathcal{P}_{\psi}(\varphi, \mathcal{C})=\inf \left\{\beta \in \mathbb{R}: \mathcal{P}_{1}(\varphi-\beta \psi, \mathcal{C}) \leq 0\right\}$

Remark 2.9. In general only " $\geq$ " holds as shown in Corollary 2.8 In the following trivial example we have " $>$ ". Consider $\Sigma$ with state space $\mathbb{N}$, incidence matrix $A_{i j}=\delta_{i j}$, and the potential $\psi(\omega)=\log \left(\omega_{1}\right)$. Then we have $\mathcal{P}_{\psi}\left(0, \Sigma^{*}\right)=\infty$, whereas inf $\left\{\beta \in \mathbb{R}: \mathcal{P}_{1}\left(-\beta \psi, \Sigma^{*}\right) \leq 0\right\}=1$.

In the next two corollaries we give sufficient conditions for the $\psi$-induced pressure to be given by the pseudo-inverse of the 1-induced pressure.

Corollary 2.10. For potential functions $\varphi, \psi: \Sigma \rightarrow \mathbb{R}$ with $\psi \geq 0$, such that the map $\beta \mapsto \mathcal{P}_{1}(\varphi-\beta \psi, \mathcal{C})$ is strictly decreasing on $\operatorname{int}\left\{\beta: \mathcal{P}_{1}(\varphi-\beta \psi, \mathcal{C})<\infty\right\}$ we have

$$
\mathcal{P}_{\psi}(\varphi, \mathcal{C})=\inf \left\{\beta \in \mathbb{R}: \mathcal{P}_{1}(\varphi-\beta \psi, \mathcal{C}) \leq 0\right\}=\sup \left\{\beta \in \mathbb{R}: \mathcal{P}_{1}(\varphi-\beta \psi, \mathcal{C}) \geq 0\right\} .
$$

In particular, this is the case if $\psi \geq c>0$. Another condition is also satisfied if $\mathcal{C}=\Sigma^{*}$, the potentials $\varphi, \psi$ are Hölder continuous, the incidence matrix of $\Sigma$ is finitely irreducible and $\psi^{-1}(\{0\})$ is at most countable.

Proof. Since the map $\beta \mapsto \mathcal{P}_{1}(\varphi-\beta \psi, \mathcal{C})$ is strictly decreasing on the set int $\left\{\beta: \mathcal{P}_{1}(\varphi-\beta \psi, \mathcal{C})<\infty\right\}$, we conclude that

$$
\begin{aligned}
\inf \left\{\beta \in \mathbb{R}: \mathcal{P}_{1}(\varphi-\beta \psi, \mathcal{C}) \leq 0\right\} & =\inf \left\{\beta \in \mathbb{R}: \mathcal{P}_{1}(\varphi-\beta \psi, \mathcal{C})<0\right\} \\
& =\sup \left\{\beta \in \mathbb{R}: \mathcal{P}_{1}(\varphi-\beta \psi, \mathcal{C}) \geq 0\right\}
\end{aligned}
$$

Since

$$
\begin{aligned}
& \inf \left\{\beta \in \mathbb{R}: \mathcal{P}_{1}(\varphi-\beta \psi, \mathcal{C})\right.<0\} \\
& \geq \inf \left\{\beta \in \mathbb{R}: \limsup _{T \rightarrow \infty} \sum_{\substack{\omega \in \mathcal{C} \\
T<S_{\omega} \psi}} \exp \left(S_{\omega} \varphi-\beta S_{\omega} \psi\right)<\infty\right\}
\end{aligned}
$$

the claim follows from Theorem 2.4 and Corollary 2.8

For $\psi \geq c>0$ the map $\beta \mapsto \mathcal{P}_{1}(\varphi-\beta \psi, \mathcal{C})$ is strictly decreasing on the set int $\left\{\beta: \mathcal{P}_{1}(\varphi-\beta \psi, \mathcal{C})<\infty\right\}$. If $\varphi$ and $\psi$ are Hölder continuous, $\psi \geq 0$ and the 
incidence matrix of $\Sigma$ is finitely irreducible, then we have by [RSU08] that $\beta \mapsto$ $\mathcal{P}_{1}\left(\varphi-\beta \psi, \Sigma^{*}\right)$ is real analytic on $\operatorname{int}\left\{\beta: \mathcal{P}_{1}\left(\varphi-\beta \psi, \Sigma^{*}\right)<\infty\right\}$ and

$$
\left.\frac{\partial}{\partial \beta} \mathcal{P}_{1}\left(\varphi-\beta \psi, \Sigma^{*}\right)\right|_{\beta_{0}}=-\int \psi d \mu_{\varphi-\beta_{0} \psi}<0,
$$

where $\mu_{\varphi-\beta_{0} \psi}$ denotes the unique invariant Gibbs measure of $\varphi-\beta_{0} \psi$, which has no atoms.

Remark 2.11. If $\Sigma$ is a subshift over a finite alphabet, i.e. $\Sigma \subset \Sigma_{n}:=\{1, \ldots, n\}^{\mathbb{N}}$ for some $n \in \mathbb{N}$, and $\psi>0$ then the map $\beta \mapsto \mathcal{P}_{1}(\varphi-\beta \psi, \mathcal{C})$ is a strictly decreasing continuous map on $\mathbb{R}$. Hence we conclude that $\mathcal{P}_{\psi}(\varphi, \mathcal{C})$ is its unique zero, i.e. $\mathcal{P}_{1}\left(\varphi-\mathcal{P}_{\psi}(\varphi, \mathcal{C}) \psi, \mathcal{C}\right)=0$.

The next Corollary shows that the exhausting principle implies, that $\mathcal{P}_{\psi}(\varphi, \mathcal{C})$ is given by the pseudo inverse of the 1-induced pressure. For ease of notation let $C_{\Sigma, \sigma}$ denote the set of compact $\sigma$-invariant subsets of $\Sigma$.

Corollary 2.12. For potential functions $\varphi, \psi \in C(\Sigma, \mathbb{R})$ satisfying $\psi>0$ and such that the exhausting principle holds for $(\psi, \varphi, \mathcal{C})$ we have

$$
\mathcal{P}_{\psi}(\varphi, \mathcal{C})=\inf \left\{\beta \in \mathbb{R}: \mathcal{P}_{1}(\varphi-\beta \psi, \mathcal{C}) \leq 0\right\}
$$

Proof. By the first part of Corollary 2.8 we only have to prove " $\leq$ ": For every $K \in C_{\Sigma, \sigma}$ we have that $\psi_{\mid K}$ is bounded away from zero and hence by the second part of Corollary 2.8 and Proposition 2.2 (1) we have

$$
\begin{aligned}
\mathcal{P}_{\psi, K}(\varphi, \mathcal{C}) & =\inf \left\{\beta \in \mathbb{R}: \mathcal{P}_{1, K}(\varphi-\beta \psi, \mathcal{C}) \leq 0\right\} \\
& \leq \inf \left\{\beta \in \mathbb{R}: \mathcal{P}_{1}(\varphi-\beta \psi, \mathcal{C}) \leq 0\right\} .
\end{aligned}
$$

Since the exhausting principle holds for $(\psi, \varphi, \mathcal{C})$ we know that there exists a sequence $\left(K_{n}\right)_{n \in \mathbb{N}} \in\left(C_{\Sigma, \sigma}\right)^{\mathbb{N}}$, such that $\lim _{n \rightarrow \infty} \mathcal{P}_{\psi, K_{n}}(\varphi, \mathcal{C})=\mathcal{P}_{\psi}(\varphi, \mathcal{C})$. Hence we conclude

$$
\mathcal{P}_{\psi}(\varphi, \mathcal{C}) \leq \inf \left\{\beta \in \mathbb{R}: \mathcal{P}_{1}(\varphi-\beta \psi, \mathcal{C}) \leq 0\right\}
$$

\section{LOOP SPACES}

In this section we introduce the $\mathcal{C}$-loop space for collections $\mathcal{C} \subset \Sigma^{*}$ satisfying certain inducing properties.

In the first part we motivate the construction and prove basic properties. In the second part we investigate the question under which assumptions we have, that two collections $\mathcal{C}^{\prime}$ and $\mathcal{C}$ give rise to the same value of the induced pressure. We will show that this question is closely linked to a certain exhausting principle. We will introduce dynamical group extensions to apply this new insights to characterise amenability of the underlying group in terms of the induced pressure. The third part of this section is devoted to a detailed comparison of the classical pressure and the Gurevič pressure; we also obtain a variational principle for the generalised Gurevič pressure. 
3.1. Construction and basic properties of loop spaces . The following definition will be crucial for the construction of loop spaces.

Definition 3.1. We say that $\mathcal{C}$ is closed under concatenations, if for any $\omega_{1}, \omega_{2} \in \mathcal{C}$ satisfying $\omega_{1} \omega_{2} \in \Sigma^{*}$ we have $\omega_{1} \omega_{2} \in \mathcal{C}$.

We say that $\mathcal{C}$ has the refinement property, if for $\omega_{1} \omega_{2} \in \mathcal{C}$ and $\omega_{2} \omega_{3} \in \mathcal{C}$ we have $\omega_{2} \in \mathcal{C}$.

If $\mathcal{C}$ is both closed under concatenations and has the refinement property then we say that $\mathcal{C}$ is representable by loops.

In fact, this representation is given in the following way. For a family $\mathcal{C} \subset \Sigma^{*}$ which is representable by loops we introduce the set of simple $\mathcal{C}$-loops as

$$
\mathcal{C}^{\text {smpl }}:=\left\{C \in \mathcal{C}: \nexists C_{1}, C_{2} \in \mathcal{C}: C=C_{1} C_{2}\right\} .
$$

We define the $\mathcal{C}$-loop space

$$
\widetilde{\Sigma}_{\mathcal{C}}:=\Sigma_{\mathcal{C}^{\text {smpl }}}:=\left\{\omega \in\left(\mathcal{C}^{\text {smpl }}\right)^{\mathbb{N}}: \forall i \in \mathbb{N}: \omega_{i} \omega_{i+1} \in \mathcal{C}\right\}
$$

which is a subshift of finite type over the alphabet $\mathcal{C}^{\text {smpl }}$. Let $\widetilde{\sigma}$ denote the corresponding shift dynamic on $\widetilde{\Sigma}_{\mathcal{C}}$ and let $\widetilde{d}_{\alpha}$ denote the corresponding metric on $\widetilde{\Sigma}_{\mathcal{C}}$

Since $\mathcal{C}$ is closed under concatenations there is a canonical map $\left(\widetilde{\Sigma}_{\mathcal{C}}\right)^{*} \rightarrow \mathcal{C}$ which we denote by $\widetilde{\omega} \mapsto \omega$. By definition of the simple elements this map is surjective and since $\mathcal{C}$ has the refinement property it is also injective.

The injection $\iota:\left(\widetilde{\Sigma}_{\mathcal{C}}, \widetilde{d}_{\alpha}\right) \hookrightarrow\left(\Sigma, d_{\alpha}\right)$ is Lipschitz continuous. We will also indicate this map by omitting the tilde, i.e. we simply write $\omega$ for $\iota(\widetilde{\omega})$.

For a function $f: \Sigma \rightarrow \mathbb{R}$ we define its induced version

$$
\widetilde{f}: \widetilde{\Sigma}_{\mathcal{C}} \rightarrow \mathbb{R} \text { by } \widetilde{f}(\widetilde{\omega}):=S_{\left|\omega_{1}\right|} f(\omega),
$$

where $\widetilde{\omega}:=\left(\widetilde{\omega}_{1}, \widetilde{\omega}_{2}, \ldots\right)$. Let us verify that if $f: \Sigma \rightarrow \mathbb{R}$ is $\alpha$-Hölder continuous then also $\tilde{f}: \widetilde{\Sigma}_{\mathcal{C}} \rightarrow \mathbb{R}$ is $\alpha$-Hölder continuous. This is a consequence of (2.2), since for $\widetilde{\omega}, \widetilde{\omega}^{\prime} \in \widetilde{\Sigma}_{\mathcal{C}}$ with $\widetilde{\omega} \wedge \widetilde{\omega}^{\prime} \geq 1$ we have

$$
\begin{aligned}
\left|\tilde{f}(\widetilde{\omega})-\widetilde{f}\left(\widetilde{\omega}^{\prime}\right)\right| & =\left|S_{\left|\omega_{1}\right|} f(\omega)-S_{\left|\omega_{1}\right|} f\left(\omega^{\prime}\right)\right| \\
& \leq \frac{V_{\alpha}(f)}{e^{\alpha}-1} d_{\alpha}\left(\sigma^{\left|\omega_{1}\right|}(\omega), \sigma^{\left|\omega_{1}\right|}\left(\omega^{\prime}\right)\right) \\
& =\frac{V_{\alpha}(f)}{e^{\alpha}-1} d_{\alpha}\left(\iota(\widetilde{\sigma}(\widetilde{\omega})), \iota\left(\widetilde{\sigma}\left(\widetilde{\omega}^{\prime}\right)\right)\right) \\
& \leq \frac{V_{\alpha}(f)}{e^{\alpha}-1} \widetilde{d}_{\alpha}\left(\widetilde{\omega}, \widetilde{\omega}^{\prime}\right) .
\end{aligned}
$$

Hence, (2.1) is satisfied with $V_{\alpha}(\widetilde{f}):=e^{\alpha} \frac{V_{\alpha}(f)}{e^{\alpha}-1}$ and consequently $\widetilde{f}$ is $\alpha$-Hölder continuous. It is important to note that Hölder continuity of $f$ is a sufficient, but not a necessary condition for the induced version $\widetilde{f}$ to be Hölder continuous.

The next Theorem shows that the $\psi$-induced pressure is invariant under inducing. Although straightforward to prove, it is of structural importance for studying loop spaces. 
Theorem 3.2 (Invariance under Inducing). Let $\varphi, \psi: \Sigma \rightarrow \mathbb{R}$ be Hölder continuous satisfying $\psi>0$ and let $\mathcal{C}$ be representable by loops. Then for $\mathcal{C}^{\prime} \subset \mathcal{C}$ and the canonically associated set $\widetilde{\mathcal{C}}^{\prime} \subset\left(\widetilde{\Sigma}_{\mathcal{C}}\right)^{*}$ we have

$$
\mathcal{P}_{\psi}\left(\varphi, \mathcal{C}^{\prime}\right)=\mathcal{P}_{\widetilde{\psi}}\left(\widetilde{\varphi}, \widetilde{\mathcal{C}}^{\prime}\right)
$$

Proof. This is a consequence of the one-to-one correspondence between elements of $\mathcal{C}^{\prime}$ and $\widetilde{\mathcal{C}^{\prime}}$ combined with the bounded distortion property for $\varphi$ and $\psi$ (see Remark 2.1).

Let us discuss the basic example, which motivates the constructions.

Example 3.3 (Gurevič pressure). For an irreducible subshift of finite type $\Sigma$ we consider the set $\mathcal{C}:=\Sigma_{a}^{\text {per }}$ for a fixed element $a \in I$. Clearly, $\Sigma_{a}^{\text {per }}$ is closed under concatenations and has the refinement property. The $\Sigma_{a}^{\text {per }}$-loop space is the full shift over the alphabet $\Sigma_{a}^{\mathrm{smpl}}$, which we denote by $\widetilde{\Sigma}_{a}$. Let $\varphi: \Sigma \rightarrow \mathbb{R}$ be a Hölder continuous potential. As a first application of the invariance under inducing we express the Gurevič pressure via the classical pressure on the corresponding loop space. By Theorem 3.2 and Corollary 2.10 we have

$$
\begin{aligned}
\mathcal{P}_{1}\left(\varphi, \Sigma_{a}^{\text {per }}\right) & =\mathcal{P}_{\widetilde{1}}\left(\widetilde{\varphi}, \widetilde{\Sigma}_{a}^{*}\right) \\
& =\inf \left\{\beta \in \mathbb{R}: \mathcal{P}_{1}\left(\widetilde{\varphi}-\beta \widetilde{1}, \widetilde{\Sigma}_{a}^{*}\right) \leq 0\right\} \\
& =\sup \left\{\beta \in \mathbb{R}: \mathcal{P}_{1}\left(\widetilde{\varphi}-\beta \widetilde{1}, \widetilde{\Sigma}_{a}^{*}\right) \geq 0\right\} .
\end{aligned}
$$

Next, we consider $\mathcal{P}_{1}\left(\widetilde{\varphi}, \widetilde{\Sigma}_{a}^{*}\right)$, which is equal to the classical pressure of $\widetilde{\varphi}$ with respect to the induced dynamical system $\left(\iota\left(\widetilde{\Sigma}_{a}\right), \sigma^{*}\right)$ with $\sigma^{*}=\sigma^{T}$, where $T(\omega):=\inf \left\{n \geq 1: \sigma^{n}(\omega) \in[a]\right\}$. Since for $\psi:=\mathbb{1}_{[a]}$ on $\Sigma$ we have $\widetilde{\psi}=1$ on $\widetilde{\Sigma}_{a}$ the invariance under inducing implies

$$
\mathcal{P}_{1}\left(\widetilde{\varphi}, \widetilde{\Sigma}_{a}^{*}\right)=\mathcal{P}_{\mathbb{1}_{[a]}}\left(\varphi, \Sigma_{a}^{\text {per }}\right),
$$

which motivates the term $\psi$-induced pressure.

In the following example we consider the geometric potential of the harmonic $\alpha$ Farey map introduced in [KMS10]. We make use of the invariance under inducing from Theorem 3.2 to determine the pressure.

Example 3.4 ( $\alpha$-Farey Map). As an example let us study the harmonic $\alpha$-Farey map $F$ defined in [KMS10], since for this map all the important quantities can be calculated explicitly. The map $F:[0,1] \rightarrow[0,1]$ is uniquely determined by the property of being linear on $I_{n}:=(1 /(n+1), 1 / n], n \in \mathbb{N}$, such that

$$
F(0)=0, \quad F\left(\frac{1}{n+1}\right)=\frac{1}{n}, n \geq 1, \quad F(1)=0 .
$$

One immediately verifies that the geometric potential $\psi:=\log \left|F^{\prime}\right|$ is positive but not bounded away from 0 . Furthermore, the family of sets $\left(I_{n}\right)$ defines a Markov partition and with respect to this partition $F$ is conjugated to the renewal shift 
with state space $\mathbb{N}$ and root 1 , which we denote by $\Sigma$. Notice, that $\Sigma$ is mixing but not finitely irreducible.

We prove that $\mathcal{P}_{1}\left(-\beta \psi, \Sigma_{1}^{\text {per }}\right)$ is strictly greater than zero for $\beta<1$ and equal to zero for $\beta \geq 1$. Furthermore, $\mathcal{P}_{1}\left(0, \Sigma_{1}^{\text {per }}\right)=\log 2$. We also show that $P_{\psi}\left(0, \Sigma_{1}^{\text {per }}\right)=$ 1 , which implies that $P_{\psi}\left(0, \Sigma_{1}^{\text {per }}\right)$ is given by the pseudo inverse of the 1 -induced pressure. Furthermore, $\sup \left\{\beta \in \mathbb{R}: \mathcal{P}_{1}\left(-\beta \psi, \Sigma_{1}^{\text {per }}\right) \geq 0\right\}=\infty$.

In order to determine $\mathcal{P}_{1}\left(-\beta \psi, \Sigma_{1}^{\text {per }}\right)$ for $\beta \in \mathbb{R}$ we consider the $\Sigma_{1}^{\text {per }}$-loop space denoted by $\widetilde{\Sigma}_{1}$. We have $\widetilde{\Sigma}_{1}=\left(\Sigma_{1}^{\text {smpl }}\right)^{\mathbb{N}}$ and we abbreviate $(1, k, k-1, \ldots, 2) \in$ $\Sigma_{1}^{\mathrm{smpl}}$ by $\widetilde{k} \in \mathbb{N}$. Observe that $\psi_{\mid[k]}=\log \left(\frac{k+1}{k-1}\right)$ for $k \geq 2$ and $\psi_{\mid[1]}=\log 2$ and hence, $\widetilde{\psi}_{\mid[\tilde{k}]}=\log (k(k+1))$ for $k \in \mathbb{N}$.

By the invariance under inducing (Theorem 3.2) we have for $\beta \in \mathbb{R}$

$$
\mathcal{P}_{1}\left(-\beta \psi, \Sigma_{1}^{\text {per }}\right)=\mathcal{P}_{\widetilde{1}}\left(-\beta \widetilde{\psi}, \widetilde{\Sigma}_{1}^{*}\right)=\inf \left\{t \in \mathbb{R}: \mathcal{P}_{1}\left(-\beta \widetilde{\psi}-t \widetilde{1}, \widetilde{\Sigma}_{1}^{*}\right) \leq 0\right\},
$$

where $\widetilde{1}_{\mid[\tilde{k}]}=k$ for $k \in \mathbb{N}$. We have

$$
\mathcal{P}_{1}\left(-\beta \widetilde{\psi}-t \widetilde{1}, \widetilde{\Sigma}_{1}^{*}\right)=\log \sum_{k \in \mathbb{N}}(k(k+1))^{-\beta} e^{-t k} .
$$

Clearly, for every $\beta \leq 1$ there exists a unique $t(\beta)$ such that

$$
\mathcal{P}_{1}\left(-\beta \widetilde{\psi}-t(\beta) \widetilde{1}, \widetilde{\Sigma}_{1}^{*}\right)=0 .
$$

In particular, we have $t(0)=\log 2, t(1)=0$ and $t(\beta)>0$ for $\beta<1$. For $\beta>1$ we have that $\mathcal{P}_{1}\left(-\beta \widetilde{\psi}-t \widetilde{1}, \widetilde{\Sigma}_{1}^{*}\right)$ is strictly less than zero for $t=0$ and is equal to infinity for $t<0$, hence inf $\left\{t \in \mathbb{R}: \mathcal{P}_{1}\left(-\beta \widetilde{\psi}-t \widetilde{1}, \widetilde{\Sigma}_{1}^{*}\right) \leq 0\right\}=0$ for every $\beta>1$. Furthermore, we have

$$
P_{\psi}\left(0, \Sigma_{1}^{\text {per }}\right)=P_{\widetilde{\psi}}\left(0, \widetilde{\Sigma}_{1}^{*}\right)=1
$$

since by the above calculation $\mathcal{P}_{1}\left(-\widetilde{\psi}, \widetilde{\Sigma}_{1}^{*}\right)=0$.

3.2. Exhausting principles and group extensions. In order to relate the induced pressure with respect to different collections $\mathcal{C} \subset \Sigma^{*}$ it will important to introduce mixing properties also with respect to such collections.

Definition 3.5. We say that $\mathcal{C}$ is irreducible, if for $C_{1}, C_{2} \in \mathcal{C}$ there exists $D \in$ $\Sigma^{*} \cup\{\emptyset\}$ such that $C_{1} D C_{2} \in \mathcal{C}$. We say that $\mathcal{C}$ is finitely irreducible, if there exists a finite set $\Lambda \subset \Sigma^{*}$, such that for $C_{1}, C_{2} \in \mathcal{C}$ there exists $D \in \Lambda \cup\{\emptyset\}$ satisfying $C_{1} D C_{2} \in \mathcal{C}$.

Remark 3.6. For $\mathcal{C}=\Sigma^{*}$ the definition coincides with the usual definition of (finite) irreducibility of the incidence matrix of $\Sigma$.

We also need the following notions of embeddability. 
Definition 3.7. Let $\mathcal{C}^{\prime}, \mathcal{C} \subset \Sigma^{*}$. We say that $\mathcal{C}$ is finitely embeddable into $\mathcal{C}^{\prime}$, if there exists a finite set $\Lambda \subset \Sigma^{*}$ such that

$$
\forall \omega \in \mathcal{C} \exists \tau_{1}, \tau_{2} \in \Lambda: \tau_{1} \omega \tau_{2} \in \mathcal{C}^{\prime} .
$$

We say that $\mathcal{C}$ is compactly finitely embeddable into $\mathcal{C}^{\prime}$, if $\mathcal{C} \cap K^{*}$ is finitely embeddable into $\mathcal{C}^{\prime}$ for all compact $\sigma$-invariant subsets $K \subset \Sigma$.

Lemma 3.8. Let $\mathcal{C}^{\prime}, \mathcal{C} \subset \Sigma^{*}$ and $\varphi, \psi: \Sigma \rightarrow \mathbb{R}$ be Hölder continuous, $\psi \geq 0$. If $\mathcal{C}$ is finitely embeddable into $\mathcal{C}^{\prime}$ then

$$
\mathcal{P}_{\psi}(\varphi, \mathcal{C}) \leq \mathcal{P}_{\psi}\left(\varphi, \mathcal{C}^{\prime}\right)
$$

Proof. For the proof it is sufficient to find a constant $C>0$, such that for all $T>0$ large and $\beta \in \mathbb{R}$ we have

$$
\sum_{\substack{\omega \in \mathcal{C} \\ T<S_{\omega} \psi}} \exp S_{\omega}(\varphi-\beta \psi) \leq C \sum_{\substack{\omega \in \mathcal{C}^{\prime} \\ T<S_{\omega} \psi}} \exp S_{\omega}(\varphi-\beta \psi) .
$$

Then the claim would follow by Theorem 2.4 .

In fact, let $\Lambda \subset \Sigma^{*}$ be the finite set witnessing the finitely embeddability condition. Let $k$ denote the maximal word length of the elements in $\Lambda$. Since $\varphi$ and $\psi$ are Hölder continuous we may choose $S_{\omega}(\varphi-\beta \psi):=\inf _{\tau \in[\omega]} S_{|\omega|}(\varphi-\beta \psi)(\tau)$ in the definition of the induced pressure. With $m:=\min _{\tau \in \Lambda} S_{\tau}(\varphi-\beta \psi)$ we have

$$
\mathrm{e}^{2 m} \sum_{\substack{\omega \in \mathcal{C} \\ T<S_{\omega} \psi}} \exp S_{\omega}(\varphi-\beta \psi) \leq \sum_{\substack{\omega \in \mathcal{C} \\ T<S_{\omega} \psi}} \exp \left(S_{\tau_{1}(\omega) \omega \tau_{2}(\omega)}(\varphi-\beta \psi)\right)
$$

Using this estimate and the fact that the map $\omega \mapsto \tau_{1}(\omega) \omega \tau_{2}(\omega)$ is at most $(2 k)$ to-1 we finally conclude

$$
\sum_{\substack{\omega \in \mathcal{C} \\ T<S_{\omega} \psi}} \exp S_{\omega}(\varphi-\beta \psi) \leq 2 k \mathrm{e}^{-2 m} \sum_{\substack{\omega \in \mathcal{C}^{\prime} \\ T<S_{\omega} \psi}} \exp \left(S_{\tau_{1}(\omega) \omega \tau_{2}(\omega)}(\varphi-\beta \psi)\right) .
$$

Remark 3.9. If in Lemma 3.8 we have additionally $\mathcal{C}^{\prime} \subset \mathcal{C}$ then

$$
\mathcal{P}_{\psi}(\varphi, \mathcal{C})=\mathcal{P}_{\psi}\left(\varphi, \mathcal{C}^{\prime}\right)
$$

Subsequently, the following notation will be useful. For an arbitrary Markov shift $\Sigma$ we let $\pi_{j}: \Sigma \rightarrow I$ denote the projection on the $j$-th coordinate, i.e. $\pi_{j}(\omega)=\omega_{j}$.

The next Lemma shows that the exhausting principle for the induced version on the associated loop space implies the exhausting principle for the original system.

Lemma 3.10. Let $\varphi, \psi: \Sigma \rightarrow \mathbb{R}$ be Hölder continuous, $\psi \geq 0$. Assume that $\mathcal{C}$ is representable by loops. If $(\widetilde{\psi}, \widetilde{\varphi}, \widetilde{\mathcal{C}})$ satisfies the exhausting principle then also $(\psi, \varphi, \mathcal{C})$ satisfies the exhausting principle.

Proof. For arbitrary $\epsilon>0$ we find a compact $\widetilde{\sigma}$-invariant subset $\widetilde{K} \subset \widetilde{\Sigma}_{\mathcal{C}}$, such that

$$
\mathcal{P}_{\widetilde{\psi}}(\widetilde{\varphi}, \widetilde{\mathcal{C}})-\epsilon \leq \mathcal{P}_{\widetilde{\psi}, \widetilde{K}}(\widetilde{\varphi}, \widetilde{\mathcal{C}})=\mathcal{P}_{\widetilde{\psi}}\left(\widetilde{\varphi}, \widetilde{\mathcal{C}} \cap \widetilde{K}^{*}\right),
$$

where the last equality follows by Remark 2.1. 
Since $\widetilde{K}$ is compact we have that $\widetilde{I}_{K}:=\pi_{1}(\widetilde{K}) \subset \mathcal{C}^{\text {smpl }}$ is compact and hence finite. The $\widetilde{\sigma}$-invariance of $\widetilde{K}$ implies that we have $\pi_{n}(\widetilde{K})=\pi_{1}\left(\widetilde{\sigma}^{n} \widetilde{K}\right)=\pi_{1}(\widetilde{K}) \subset \widetilde{I}_{K}$ and therefore $\widetilde{K} \subset\left(\widetilde{I}_{K}\right)^{\mathbb{N}}$. By Proposition 2.2 (11) and Theorem 3.2 we have

$$
\mathcal{P}_{\widetilde{\psi}}\left(\widetilde{\varphi}, \widetilde{\mathcal{C}} \cap \widetilde{K}^{*}\right) \leq \mathcal{P}_{\widetilde{\psi}}\left(\widetilde{\varphi}, \widetilde{\mathcal{C}} \cap \widetilde{I}_{K}^{*}\right)=\mathcal{P}_{\psi}\left(\varphi, \mathcal{C} \cap\left\{\omega \in \Sigma^{*}: \widetilde{\omega}_{i} \in \widetilde{I}_{K}\right\}\right)
$$

Next, we define $I_{K}$ as the (finite) set of all elements in $I$, which are needed to represent the elements of $\widetilde{I}_{K}$. Then $K:=\left(I_{K}\right)^{\mathbb{N}} \cap \Sigma$ is compact and $\sigma$-invariant. By Proposition 2.2 (1) and Remark 2.1

$$
\mathcal{P}_{\psi}\left(\varphi, \mathcal{C} \cap\left\{\omega \in \Sigma^{*}: \widetilde{\omega}_{i} \in \widetilde{I}_{K}\right\}\right) \leq \mathcal{P}_{\psi}\left(\varphi, \mathcal{C} \cap K^{*}\right)=\mathcal{P}_{\psi, K}(\varphi, \mathcal{C}) .
$$

Finally combining Theorem 3.2 with the above estimates we conclude that $\mathcal{P}_{\psi}(\varphi, \mathcal{C})-$ $\epsilon=\mathcal{P}_{\widetilde{\psi}}(\widetilde{\varphi}, \widetilde{\mathcal{C}})-\epsilon \leq \mathcal{P}_{\psi, K}(\varphi, \mathcal{C})$

Remark 3.11. We would like to point out that the conditions of Lemma 3.10 in particular imply that by Corollary 2.12 we have

$$
\mathcal{P}_{\psi}(\varphi, \mathcal{C})=\inf \left\{\beta \in \mathbb{R}: \mathcal{P}_{1}(\varphi-\beta \psi, \mathcal{C}) \leq 0\right\},
$$

whereas in general $\mathcal{P}_{\psi}(\varphi, \mathcal{C})$ is not equal to $\sup \left\{\beta \in \mathbb{R}: \mathcal{P}_{1}(\varphi-\beta \psi, \mathcal{C}) \geq 0\right\}$ as seen in Example 3.4.

Next Theorem considers subsystems $\mathcal{C}^{\prime} \subset \mathcal{C}$ with $\mathcal{C}$ compactly finitely embeddable into $\mathcal{C}^{\prime}$. Under the condition that $\mathcal{C}^{\prime}$ is finitely irreducible we prove that $\mathcal{P}_{\psi}(\varphi, \mathcal{C})=$ $\mathcal{P}_{\psi}\left(\varphi, \mathcal{C}^{\prime}\right)$ is equivalent to the exhausting principle for $(\psi, \varphi, \mathcal{C})$.

Theorem 3.12. Let $\varphi, \psi: \Sigma \rightarrow \mathbb{R}$ be Hölder continuous with $\psi>0$. Let $\mathcal{C}^{\prime} \subset \mathcal{C} \subset$ $\Sigma^{*}$ and suppose that $\mathcal{C}^{\prime}$ is finitely irreducible and representable by loops, and that $\mathcal{C}$ be compactly finitely embeddable into $\mathcal{C}^{\prime}$. Then

$$
\sup _{K \in C_{\Sigma, \sigma}} \mathcal{P}_{\psi, K}(\varphi, \mathcal{C})=\mathcal{P}_{\psi}\left(\varphi, \mathcal{C}^{\prime}\right)
$$

In particular, we have

$$
(\psi, \varphi, \mathcal{C}) \text { satisfies the exhausting principle } \Longleftrightarrow \mathcal{P}_{\psi}(\varphi, \mathcal{C})=\mathcal{P}_{\psi}\left(\varphi, \mathcal{C}^{\prime}\right) .
$$

Proof. Since $\mathcal{C}^{\prime}$ is finitely irreducible and $\widetilde{\varphi}, \widetilde{\psi}$ are Hölder continuous, making a similar calculation as in the proof of [MU03, Theorem 2.1.5] we readily find that $\left(1, \widetilde{\varphi}-\beta \widetilde{\psi}, \widetilde{\Sigma}_{\mathcal{C}^{\prime}}^{*}\right)$ satisfies the exhausting principle for each $\beta \in \mathbb{R}$. Next, we show that also $\left(\widetilde{\psi}, \widetilde{\varphi}, \widetilde{\Sigma}_{\mathcal{C}^{\prime}}^{*}\right)$ satisfies the exhausting principle. For this, let $\delta>0$. By Corollary 2.10 we have $\mathcal{P}_{\widetilde{\psi}}\left(\widetilde{\varphi}, \widetilde{\Sigma}_{\mathcal{C}^{\prime}}^{*}\right)=\inf \left\{\beta \in \mathbb{R}: \mathcal{P}_{1}\left(\widetilde{\varphi}-\beta \widetilde{\psi}, \widetilde{\Sigma}_{\mathcal{C}^{\prime}}^{*}\right) \leq 0\right\}$ and hence

$$
\mathcal{P}_{1}\left(\widetilde{\varphi}-\left(\mathcal{P}_{\widetilde{\psi}}\left(\widetilde{\varphi}, \widetilde{\Sigma}_{\mathcal{C}^{\prime}}^{*}\right)-\delta\right) \widetilde{\psi}, \widetilde{\Sigma}_{\mathcal{C}^{\prime}}^{*}\right)>0 .
$$

Since the exhausting principle holds for $\left(1, \widetilde{\varphi}-\beta \widetilde{\psi}, \widetilde{\Sigma}_{\mathcal{C}^{\prime}}^{*}\right)$ with $\beta \in \mathbb{R}$, we find a compact $\widetilde{\sigma}$-invariant subset $\widetilde{K} \subset \widetilde{\Sigma}_{\mathcal{C}^{\prime}}$, such that

$$
\mathcal{P}_{1, \widetilde{K}}\left(\widetilde{\varphi}-\left(\mathcal{P}_{\widetilde{\psi}}\left(\widetilde{\varphi}, \widetilde{\Sigma}_{\mathcal{C}^{\prime}}^{*}\right)-\delta\right) \widetilde{\psi}, \widetilde{\Sigma}_{\mathcal{C}^{\prime}}^{*}\right)>0
$$


Using Corollary 2.8 we get

$$
\mathcal{P}_{\widetilde{\psi}, K}\left(\widetilde{\varphi}, \mathcal{C}^{\prime}\right) \geq \inf \left\{\beta \in \mathbb{R}: \mathcal{P}_{1, \widetilde{K}}(\widetilde{\varphi}-\beta \widetilde{\psi}, \mathcal{C}) \leq 0\right\} \geq \mathcal{P}_{\widetilde{\psi}}\left(\widetilde{\varphi}, \mathcal{C}^{\prime}\right)-\delta
$$

and hence $\left(\widetilde{\psi}, \widetilde{\varphi}, \widetilde{\Sigma}_{\mathcal{C}^{\prime}}^{*}\right)$ satisfies the exhausting principle.

By Lemma 3.10 also $\left(\psi, \varphi, \mathcal{C}^{\prime}\right)$ satisfies the exhausting principle. Using this and Proposition 2.2 (1) we therefore have

$$
\sup _{K \in C_{\Sigma, \sigma}} \mathcal{P}_{\psi, K}(\varphi, \mathcal{C}) \geq \sup _{K \in C_{\Sigma, \sigma}} \mathcal{P}_{\psi, K}\left(\varphi, \mathcal{C}^{\prime}\right)=\mathcal{P}_{\psi}\left(\varphi, \mathcal{C}^{\prime}\right)
$$

For the reverse inequality notice that $\mathcal{C} \cap K^{*}$ is finitely embeddable into $\mathcal{C}^{\prime}$ for $K \in C_{\Sigma, \sigma}$, which by Lemma 3.8 implies that

$$
\mathcal{P}_{\psi, K}(\varphi, \mathcal{C}) \leq \mathcal{P}_{\psi}\left(\varphi, \mathcal{C}^{\prime}\right)
$$

Corollary 3.13. For Hölder continuous functions $\varphi, \psi$ with $\psi>0$ and $\mathcal{C} \subset \Sigma^{*}$ finitely irreducible and representable by loops, we have that $(\psi, \varphi, \mathcal{C})$ satisfies the exhausting principle.

Definition 3.14. For a fixed starting set $J_{s} \subset I$ and a terminating set $J_{t} \subset I$ we denote by $\mathcal{C}=\left\{\omega \in \Sigma^{*}: \omega_{1} \in J_{s}\right\}$ the set of starting words in $J_{s}$ and by $\mathcal{C}^{\prime}=$ $\left\{\omega \in \mathcal{C}: \omega_{1} \in J_{s}, \omega_{|\omega|} \in J_{t}\right\}$ the set of bridges from $J_{s}$ to $J_{t}$.

Corollary 3.15 (Exhausting principle for bridges). Let the incidence matrix of $\Sigma$ be irreducible and let $\varphi, \psi: \Sigma \rightarrow \mathbb{R}$ be Hölder continuous, $\psi>0$. Furthermore, let $\mathcal{C}$ be the set of words starting in the non-empty and finite set $J_{s} \subset I$ and let $\mathcal{C}^{\prime}$ be the set of bridges from $J_{s}$ to the non-empty and finite set $J_{t} \subset I$. Then

$$
\sup _{K \in C_{\Sigma, \sigma}} \mathcal{P}_{\psi, K}(\varphi, \mathcal{C})=\mathcal{P}_{\psi}\left(\varphi, \mathcal{C}^{\prime}\right) .
$$

In particular, we have

$$
(\psi, \varphi, \mathcal{C}) \text { satisfies the exhausting principle } \Longleftrightarrow \mathcal{P}_{\psi}(\varphi, \mathcal{C})=\mathcal{P}_{\psi}\left(\varphi, \mathcal{C}^{\prime}\right) \text {. }
$$

Proof. We will verify the assumptions of Theorem 3.12. Clearly, $\mathcal{C}^{\prime}$ is closed under concatenations, has the refinement property and is hence representable by loops. Since $\Sigma$ is irreducible and the sets $J_{s}$ and $J_{t}$ are finite we conclude that $\mathcal{C}^{\prime}$ is finitely irreducible. It remains to show that $\mathcal{C}$ is compactly finitely embeddable into $\mathcal{C}^{\prime}$. Let $K \in C_{\Sigma, \sigma}$ be compact and $\sigma$-invariant. Repeating the arguments in the proof of Lemma 3.10 we find that $K \subset \Sigma_{N}$ for some $N \in \mathbb{N}$. Fix some $\gamma \in \mathcal{C}^{\prime}$. By the definition of $\mathcal{C}^{\prime}$ and the fact that $\Sigma$ is irreducible we find for all $\omega \in \mathcal{C} \cap K^{*}$ elements $\tau_{1}, \tau_{2} \in \Sigma^{*}$ such that $\gamma \tau_{1} \omega \tau_{2} \gamma \in \mathcal{C}^{\prime}$. In fact, $\tau_{1}, \tau_{2}$ can be taken from a finite set $\Lambda_{K} \subset \Sigma^{*}$, since the elements $\omega \in \mathcal{C} \cap K^{*}$ are constructed over the finite alphabet $\{1, \ldots, N\}$.

Example 3.16 (Simple Random Walk). By Corollary 3.13 we know that a sufficient condition for the exhausting principle to hold for $(\psi, \varphi, \mathcal{C})$ and arbitrary Hölder continuous functions $\varphi$ and $\psi>0$ is that $\mathcal{C}$ is finitely irreducible and representable by loops. This example shows that this condition is not necessary.

Let us consider

$$
\Sigma_{\mathbb{Z}}=\left\{\left(\tau_{n}, h_{n}\right) \in(\{-1,+1\} \times \mathbb{Z})^{\mathbb{N}}: h_{n}+\tau_{n}=h_{n+1}\right\}
$$


together with the starting set $J_{s}:=\{(1,0),(-1,0)\}$ and terminating set $J_{t}:=$ $\{(1,-1),(-1,1)\}$. The corresponding sets of starting words in $J_{s}$ and bridges from $J_{s}$ to $J_{t}$ are then given by

$$
\mathcal{C}:=\left\{\omega \in \Sigma_{\mathbb{Z}}^{*}: h_{1}=0\right\}, \quad \mathcal{C}^{\prime}:=\left\{\omega \in \mathcal{C}: h_{|\omega|}+\tau_{|\omega|}=0\right\} .
$$

Notice that $\Sigma_{\mathbb{Z}}$ is irreducible. It is also evident that $\mathcal{C}$ is not finitely irreducible. Nevertheless, we will show that $(1,0, \mathcal{C})$ satisfies the exhausting principle. By Corollary 3.15 this is equivalent to $\mathcal{P}_{1}(0, \mathcal{C})=\mathcal{P}_{1}\left(0, \mathcal{C}^{\prime}\right)$.

Clearly, $\mathcal{P}_{1}(0, \mathcal{C})=\log 2$ and we will prove that also $\mathcal{P}_{1}\left(0, \mathcal{C}^{\prime}\right)=\log 2$. For this note that card $\left\{\omega \in \mathcal{C}^{\prime},|\omega|=2 n\right\}=\left(\begin{array}{c}2 n \\ n\end{array}\right)$, which is by Stirling's formula comparable to $2^{2 n} n^{-1 / 2}$. Taking logarithm and dividing by $2 n$ proves the assertion.

Let us also give an alternative proof using the invariance under inducing as stated in Theorem 3.2. We have

$$
\mathcal{P}_{1}\left(0, \mathcal{C}^{\prime}\right)=\mathcal{P}_{\widetilde{1}}\left(0, \widetilde{\Sigma}_{\mathcal{C}^{\prime}}^{*}\right)=\inf \left\{t \in \mathbb{R}: \mathcal{P}_{1}\left(-\beta \widetilde{1}, \widetilde{\Sigma}_{\mathcal{C}^{\prime}}^{*}\right) \leq 0\right\}
$$

Since $\widetilde{\Sigma}_{\mathcal{C}^{\prime}}$ is a full shift,

$$
\mathcal{P}_{1}\left(-\beta \widetilde{1}, \widetilde{\Sigma}_{\mathcal{C}^{\prime}}^{*}\right)=\log \sum_{\omega \in\left(\mathcal{C}^{\prime}\right)^{\mathrm{smpl}}} \mathrm{e}^{-\beta|\omega|} .
$$

Following Kes59b] we compute

$$
\operatorname{card}\left\{\omega \in\left(\mathcal{C}^{\prime}\right)^{\mathrm{smpl}},|\omega|=2 n\right\}=\frac{2}{n}\left(\begin{array}{c}
2 n-2 \\
n-1
\end{array}\right)
$$

and using the Binomial Theorem we find for $\beta \geq \log 2$ that $\mathcal{P}_{1}\left(-\beta \widetilde{1}, \widetilde{\Sigma}_{\mathcal{C}^{\prime}}^{*}\right)$ is finite and equal to $\log \left(1-\sqrt{1-(\log 2-\beta)^{2}}\right)$. We conclude $\mathcal{P}_{1}\left(-\log 2 \cdot \widetilde{1}, \widetilde{\Sigma}_{\mathcal{C}^{\prime}}^{*}\right)=0$ and hence $\mathcal{P}_{1}\left(0, \mathcal{C}^{\prime}\right)=\log 2$.

In fact, the above example is a special case of the following general Theorem. Let $\mathbb{F}_{k}:=\left\langle g_{1}, \ldots, g_{k}\right\rangle$ denote the free group of rank $k \geq 1$ and define $I:=$ $\left\{g_{1}, \ldots, g_{k}, g_{1}^{-1}, \ldots, g_{k}^{-1}\right\}$ to be the set of symmetric generators of $\mathbb{F}_{k}$. Let $N$ be a normal subgroup of $\mathbb{F}_{k}$ and let $\pi: \mathbb{F}_{k} \rightarrow \mathbb{F}_{k} / N=$ : $G$ be the canonical factor map. Let us consider the two naturally associated Markov shifts

$$
\Sigma_{G}:=\left\{\left(\tau_{n}, h_{n}\right) \in(I \times G)^{\mathbb{N}}: h_{n} \pi\left(\tau_{n}\right)=h_{n+1}\right\}
$$

and

$$
\underline{\Sigma}_{G}:=\left\{\left(\tau_{n}, h_{n}\right) \in(I \times G)^{\mathbb{N}}: \tau_{n} \tau_{n+1} \neq \mathrm{id}, h_{n} \pi\left(\tau_{n}\right)=h_{n+1}\right\},
$$

where in the latter case we always assume $k \geq 2$ and $N \neq\{$ id $\}$.

Note, that this construction was introduced before e.g. in [KS07], where it was called the modular shift space in the context of finite index subgroups of $\mathrm{PSL}_{2}(\mathbb{Z})$. This representation is closely connected to a skew product dynamical system, since for $\left(\tau_{n}, h_{n}\right) \in \Sigma_{G}$ or $\underline{\Sigma}_{G}$ we have $h_{n}=h_{1} \pi\left(\tau_{1}\right) \cdots \pi\left(\tau_{n}\right)$ for $n \geq 2$.

Theorem 3.17 (Group extension). For $\Xi$ either equal to $\Sigma_{G}$ or $\underline{\Sigma}_{G}$, let $\mathcal{C}^{\prime} \subset \mathcal{C}$ be given by

$$
\mathcal{C}:=\left\{\omega \in \Xi: h_{1}=\mathrm{id}\right\} \quad \text { and } \mathcal{C}^{\prime}:=\left\{\omega \in \mathcal{C}: h_{|\omega|} \pi\left(\tau_{|\omega|}\right)=\mathrm{id}\right\}
$$


We have the following chain of equivalences.

$$
\begin{aligned}
G \text { is amenable } & \Longleftrightarrow(1,0, \mathcal{C}) \text { satisfies the exhausting principle } \\
& \Longleftrightarrow \mathcal{P}_{1}(0, \mathcal{C})=\mathcal{P}_{1}\left(0, \mathcal{C}^{\prime}\right) .
\end{aligned}
$$

Moreover,

$$
G \text { finite } \Longleftrightarrow \mathcal{C} \text { finite irreducible } \Longleftrightarrow \Xi \text { compact. }
$$

Remark 3.18. For $\Xi=\underline{\Sigma}_{G}$ there is a canonical one-to-one correspondence between both the sets $\mathcal{C}$ and $\mathbb{F}_{k}$, and the sets $\mathcal{C}^{\prime}$ and $N$. This allows an interpretation of our result in terms of the Poincaré exponent.

Proof. One easily verifies that the incidence matrix of $\Xi$ is irreducible. Note that $\mathcal{C}$ is in fact the set of starting words in $J_{s}:=\{(g$, id $): g \in I\}$ and $\mathcal{C}^{\prime}$ is the set of bridges from $J_{s}$ to $J_{t}:=\left\{\left(g, \pi\left(g^{-1}\right)\right): g \in I\right\}$. Hence, by Corollary 3.15 we have

$$
(1,0, \mathcal{C}) \text { satisfies the exhausting principle } \Longleftrightarrow \mathcal{P}_{1}(0, \mathcal{C})=\mathcal{P}_{1}\left(0, \mathcal{C}^{\prime}\right) \text {. }
$$

Let us first consider the case $\Xi=\Sigma_{G}$. In order to prove that this is also equivalent to $G$ being amenable we make use of Kesten's characterisation of finitely generated amenable groups ([Kes59b, Kes59a $])$. We introduce the stochastic matrix

$$
p\left(g, g^{\prime}\right):=\frac{1}{2 k} \operatorname{card}\left\{1 \leq i \leq 2 k: g \pi\left(g_{i}\right)=g^{\prime}\right\}, \quad g, g^{\prime} \in G,
$$

which defines a symmetric random walk on $G$ with the property that

$$
\mathcal{P}_{1}\left(0, \mathcal{C}^{\prime}\right)=\log (2 k)+\underset{n}{\limsup } \frac{1}{n} \log p^{(n)}(\mathrm{id}, \mathrm{id}) .
$$

By (Kes59a, Main Theorem]) we have $\lim \sup _{n} \frac{1}{n} \log p^{(n)}$ (id, id) $=0$, if and only if $G$ is amenable. Since $\mathcal{P}_{1}(0, \mathcal{C})=\log (2 k)$ the assertion follows.

Next we consider the case $\Xi=\underline{\Sigma}_{G}$ for $k \geq 2$ and $N \neq\{$ id $\}$. Clearly, in this case we have $\mathcal{P}_{1}(0, \mathcal{C})=\log (2 k-1)$. By Remark 3.18 the elements of $\mathcal{C}^{\prime}$ correspond to the elements of $N$. By Grigorchuk's cogrowth criterion (see Gri80] and also Coh82, Kes67, GdlH01]) we conclude that $\mathcal{P}_{1}\left(0, \mathcal{C}^{\prime}\right)=\log (2 k-1)$, if and only if $G$ is amenable. This finishes the proof of the first part.

The second statement of the theorem is in both cases an immediate consequence of the construction of $\Xi$ and $\mathcal{C}$. In fact, for $G$ finite, $\Xi$ is a subshift of finite type with finite state space.

Having regard to the results of Brooks in [Bro85] we expect the above characterisation of amenability to hold true for a much larger class of Hölder continuous functions $\varphi$ and $\psi>0$.

3.3. Gurevič pressure. In this section we apply our results to the Gurevič pressure (cf. [Gur69, Gur70, Sar99, Sar01, Sar03]). Recall that the Gurevič pressure is defined only for mixing subshifts of finite type and in that setting coincides with $\mathcal{P}_{1}\left(\varphi, \Sigma_{a}^{\text {per }}\right)$ for $a \in I$.

As a direct application of the invariance under inducing of Theorem 3.2, Lemma 3.10, and Corollary 2.12 we give a new description of the Gurevič pressure in terms of the classical pressure for infinite systems as investigated e.g. by Mauldin and Urbański in MU03. 
Corollary 3.19. Let $\varphi, \psi: \Sigma \rightarrow \mathbb{R}$ be Hölder continuous, $\psi>0$. Then the exhausting principle holds for $\left(\psi, \varphi, \Sigma_{a}^{\mathrm{per}}\right)$ and any $a \in I$. Furthermore, we have

$$
\begin{aligned}
\mathcal{P}_{\psi}\left(\varphi, \Sigma_{a}^{\text {per }}\right) & =\inf \left\{\beta: \mathcal{P}_{1}\left(\varphi-\beta \psi, \Sigma_{a}^{\text {per }}\right) \leq 0\right\} \\
& =\inf \left\{\beta \in \mathbb{R}: \mathcal{P}_{1}\left(\widetilde{\varphi}-\beta \widetilde{\psi}, \widetilde{\Sigma}_{a}^{\text {per }}\right) \leq 0\right\} \\
& =\sup \left\{\beta \in \mathbb{R}: \mathcal{P}_{1}\left(\widetilde{\varphi}-\beta \widetilde{\psi}, \widetilde{\Sigma}_{a}^{\text {per }}\right) \geq 0\right\} .
\end{aligned}
$$

Remark 3.20. In particular, for potentials $\psi$, such that the induced version $\widetilde{\psi}$ on

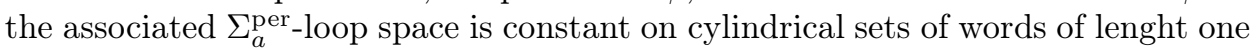

$$
\mathcal{P}_{\psi}\left(0, \Sigma_{a}^{\mathrm{per}}\right)=\inf \left\{\beta \in \mathbb{R}: \sum_{\omega \in \Sigma_{a}^{\text {smpl }}} e^{-\beta S_{\omega} \psi}<1\right\} .
$$

See also the dicussion of the special semi-flow presented in the introduction.

It is well known that the Gurevič pressure for $\psi=1$ and a mixing subshift of finite type is independent of $a \in I$. The following generalisation for $\psi$-induced pressure follows from Corollary 3.19 and Lemma 3.8, since $\Sigma_{a}^{\text {per }}$ is finitely embeddable into $\Sigma_{b}^{\text {per }}$ for $a, b \in I$.

Fact 3.21. Let $\varphi, \psi: \Sigma \rightarrow \mathbb{R}$ be Hölder continuous, $\psi>0$, and A irreducible. Then we have that $\mathcal{P}_{\psi}\left(\varphi, \Sigma_{a}^{\text {per }}\right)$ is independent of the choice of $a \in I$.

As a consequence of Proposition 2.2 (11) we have for the particular choices $\mathcal{C}=\Sigma_{a}^{\text {per }}$, $\mathcal{C}=\Sigma^{\text {per }}$ and $\mathcal{C}=\Sigma^{*}$ the following relation.

Fact 3.22. For $\varphi, \psi: \Sigma \rightarrow \mathbb{R}$ Hölder continuous, $\psi \geq 0$, we have

$$
\mathcal{P}_{\psi}\left(\varphi, \Sigma_{a}^{\text {per }}\right) \leq \mathcal{P}_{\psi}\left(\varphi, \Sigma^{\text {per }}\right) \leq \mathcal{P}_{\psi}\left(\varphi, \Sigma^{*}\right)
$$

For the following, we denote the irreducible component of $\Sigma$ containing a by $\Sigma(a):=$ $\left\{\omega \in \Sigma: a \curvearrowright \omega_{1}, \forall i \in \mathbb{N} \omega_{i} \curvearrowright a\right\}$, where $a \curvearrowright b$ means, that there exists $\tau \in \Sigma^{*}$ with $a \tau b \in \Sigma^{*}$. Theorem 3.12 provides us with a dichotomy for the Gurevič pressure and the classical pressure.

Corollary 3.23 (Classicle Pressure-Gurevič Pressure Dichotomy). Let $\varphi, \psi: \Sigma \rightarrow$ $\mathbb{R}$ be Hölder continuous with $\psi>0$. Then the following holds.

(1) $\sup _{K \in C_{\Sigma, \sigma}} \mathcal{P}_{\psi, K}\left(\varphi, \Sigma(a)^{*}\right)=\mathcal{P}_{\psi}\left(\varphi, \Sigma_{a}^{\text {per }}\right)$ for $a \in I$.

(2) $\sup _{K \in C_{\Sigma, \sigma}} \mathcal{P}_{\psi, K}\left(\varphi, \Sigma^{*}\right)=\sup _{a \in I} \mathcal{P}_{\psi}\left(\varphi, \Sigma_{a}^{\text {per }}\right)$.

In particular, if $\Sigma$ is irreducible then we have for every $a \in I$,

$$
\sup _{K \in C_{\Sigma, \sigma}} \mathcal{P}_{\psi, K}\left(\varphi, \Sigma^{*}\right)=\mathcal{P}_{\psi}\left(\varphi, \Sigma_{a}^{\text {per }}\right)
$$

and the following equivalence holds:

$\left(\psi, \varphi, \Sigma^{*}\right)$ satisfies the exhausting principle $\Longleftrightarrow \mathcal{P}_{\psi}\left(\varphi, \Sigma^{*}\right)=\mathcal{P}_{\psi}\left(\varphi, \Sigma_{a}^{\text {per }}\right)$.

Proof. ad (1): The " $\geq$ "-part follows, since $\Sigma(a)^{*} \supset \Sigma_{a}^{\text {per }}$ and $\left(\psi, \varphi, \Sigma_{a}^{\text {per }}\right)$ satisfies the exhausting principle by Corollary 3.19] For the " $\leq$ "-part recall that every $K \in C_{\Sigma, \sigma}$ is contained in a set $\Sigma_{N}$ for some $N \in \mathbb{N}$. By definition of $\Sigma(a)$ we find for every element $\omega \in \Sigma(a)^{*}$ elements $\tau_{1}, \tau_{2} \in \Sigma^{*}$ such that $\tau_{1} \omega \tau_{2} \in \Sigma_{a}^{\text {per }}$. For 
$\omega \in \Sigma(a)^{*} \cap \Sigma_{N}$ the elements $\tau_{1}, \tau_{2}$ can be chosen from a finite set. We conclude that $\Sigma(a)^{*} \cap \Sigma_{N}$ is finitely embeddable into $\Sigma_{a}^{\text {per }}$ and hence the upper bound follows by Lemma 3.8 .

ad (2): We only comment on the " $\leq$ "-part. Let $K \in C_{\Sigma, \sigma}$ with $K \subset \Sigma_{N}$ for some $N \in \mathbb{N}$. We have

$$
\mathcal{P}_{\psi, K}\left(\varphi, \Sigma^{*}\right) \leq \max _{n \in\{1, \ldots, N\}} \mathcal{P}_{\psi, \Sigma_{N}}\left(\varphi, \Sigma(n)^{*}\right) \leq \max _{n \in\{1, \ldots, N\}} \mathcal{P}_{\psi, \Sigma_{N}}\left(\varphi, \Sigma_{n}^{\text {per }}\right),
$$

where the first inequality follows by decomposition in the finitely many irreducible components and the second by an application of part (1).

Restricting to finitely irreducible systems we obtain

Corollary 3.24. Let the incidence matrix of $\Sigma$ be finitely irreducible and let $\varphi, \psi$ : $\Sigma \rightarrow \mathbb{R}$ be Hölder continuous, $\psi>0$. Then we have

$$
\mathcal{P}_{\psi}\left(\varphi, \Sigma_{a}^{\text {per }}\right)=\mathcal{P}_{\psi}\left(\varphi, \Sigma^{\text {per }}\right)=\mathcal{P}_{\psi}\left(\varphi, \Sigma^{*}\right)
$$

and

$$
\mathcal{P}_{\psi}\left(\varphi, \Sigma_{a}^{\text {per }}\right)=\inf \left\{\beta: \mathcal{P}_{1}\left(\varphi-\beta \psi, \Sigma^{*}\right) \leq 0\right\}=\sup \left\{\beta: \mathcal{P}_{1}\left(\varphi-\beta \psi, \Sigma^{*}\right) \geq 0\right\} .
$$

Next, we give an example where $\mathcal{P}_{\psi}\left(0, \Sigma_{1}^{\text {per }}\right)<\mathcal{P}_{\psi}\left(0, \Sigma^{*}\right)$ whereas $\mathcal{P}_{\psi}\left(\varphi, \Sigma_{1}^{\text {per }}\right)=$ $\mathcal{P}_{\psi}\left(\varphi, \Sigma^{*}\right)$ for some Hölder continuous potentials $\psi, \varphi: \Sigma \rightarrow \mathbb{R}$ with $\psi>0$. Necessarily, the incidence matrix in this example is not finitely irreducible.

Example 3.25. We consider again the renewal shift $\Sigma$ and the potential $\psi: \Sigma \rightarrow \mathbb{R}$ satisfying $\psi_{[[n]}=\log \left(\frac{n+1}{n-1}\right)$ for $n \geq 2$ and $\psi_{\mid[1]}=\log 2$ from Example [3.4. In Example 3.4 we proved $\mathcal{P}_{\psi}\left(0, \Sigma_{1}^{\text {per }}\right)=1$, now we consider $\mathcal{P}_{\psi}\left(0, \Sigma^{*}\right)$. We calculate that for $n, k \in \mathbb{N}$ with $1 \leq k<n$ and $\tau \in[n, n-1, \ldots, n-k+1]$

$$
S_{k} \psi(\tau)=\log ((n(n+1)) /((n-k+1)(n-k))) .
$$

We conclude that for all $T>0$ and $\beta \in \mathbb{R}$ we have $\sum_{\substack{\omega \in \Sigma^{*} \\ T<S_{\omega} \psi}} \mathrm{e}^{-\beta S_{\omega} \psi}=\infty$. Hence, by Theorem 2.4 we have $\mathcal{P}_{\psi}\left(0, \Sigma^{*}\right)=\infty$. Since $\Sigma^{*}$ is compactly finitely embeddable into $\Sigma_{1}^{\text {per }}$ we infer that $\left(\psi, 0, \Sigma^{*}\right)$ does not satisfy the exhausting principle by Theorem 3.12 ,

Next, we introduce the potential $\varphi: \Sigma \rightarrow \mathbb{R}$ given by $\varphi(\omega):=-\omega_{1}$. We verify that $\mathcal{P}_{\psi}\left(\varphi, \Sigma_{1}^{\text {per }}\right)=\mathcal{P}_{\psi}\left(\varphi, \Sigma^{*}\right)$ and hence $\left(\psi, \varphi, \Sigma^{*}\right)$ satisfies the exhausting principle. By decomposing the partition function corresponding to $\Sigma^{*}$ in a product we obtain

$$
\begin{aligned}
\sum_{\omega \in \Sigma^{*}} \mathrm{e}^{S_{\omega}(\varphi-\beta \psi)=} & \left(1+\sum_{n \geq 2} \mathrm{e}^{S_{(n, n-1, \ldots, 2)}(\varphi-\beta \psi)}\right) \sum_{\omega \in \Sigma_{1}^{\text {per }}} \mathrm{e}^{S_{\omega}(\varphi-\beta \psi)} \\
& \times\left(1+\sum_{N \geq 2} \sum_{1 \leq k<N} \mathrm{e}^{S_{(1, N, N-1, \ldots, N-k+1)}(\varphi-\beta \psi)}\right) .
\end{aligned}
$$

Using Theorem 2.4 we conclude that $\mathcal{P}_{\psi}\left(\varphi, \Sigma^{*}\right)$ is the maximum of $\mathcal{P}_{\psi}\left(\varphi, \Sigma_{1}^{\text {per }}\right)$, $\mathcal{P}_{\psi}(\varphi,\{(n, n-1, \ldots, 1): n \geq 2\})$, and

$$
\mathcal{P}_{\psi}(\varphi,\{(1, N, \ldots, N-k+1): N \geq 2,1 \leq k<N\}) .
$$


The claim then follows by showing that the latter two terms are equal to $-\infty$. In fact, since $\exp \left(S_{\omega} \varphi\right) \leq \exp \left(-\omega_{2}\right)$ we have, for every $\beta \in \mathbb{R}$, that

$$
\sum_{n \geq 2} \mathrm{e}^{S_{(n, n-1, \ldots, 1)}(\varphi-\beta \psi)} \leq \sum_{n \geq 2} \mathrm{e}^{-n+1}(n(n+1))^{-\beta}<\infty
$$

as well as

$$
\begin{aligned}
\sum_{N \geq 2} \sum_{1 \leq k<N} \mathrm{e}^{S_{(1, N, N-1, \ldots, N-k+1)} \varphi-\beta \psi} & \leq \sum_{\substack{N \geq 2\\
}} \mathrm{e}^{-N} \sum_{1 \leq k<N}\left(\frac{2 N(N+1)}{(N-k+1)(N-k)}\right)^{-\beta} \\
& <\infty
\end{aligned}
$$

We finish this section by stating a variational principle generalising results from [BI06] to our situation. This result will be crucial also for our results on the topological pressure for special semi-flows in Section 4.

Proposition 3.26 (Variational Principle). Let the incidence matrix of $\Sigma$ be irreducible and let $\varphi, \psi: \Sigma \rightarrow \mathbb{R}$ be Hölder continuous with $\psi>0$. Then we have for every $a \in I$

$$
\begin{aligned}
\mathcal{P}_{\psi}\left(\varphi, \Sigma_{a}^{\mathrm{per}}\right) & =\sup \left\{\frac{h_{\nu}(\sigma)}{\int \psi d \nu}+\frac{\int \varphi d \nu}{\int \psi d \nu}: \nu \in \mathcal{M}_{\sigma}^{1} \text { with } \varphi, \psi \in L^{1}(\nu)\right\} \\
& =\sup \left\{\frac{h_{\nu}(\sigma)}{\int \psi d \nu}+\frac{\int \varphi d \nu}{\int \psi d \nu}: \nu \in \mathcal{E}_{\sigma, c}^{1}\right\}
\end{aligned}
$$

where $\mathcal{M}_{\sigma}^{1}$ denotes the set of $\sigma$-invariant probability measures on $\Sigma$, and $\mathcal{E}_{\sigma, c}^{1} \subset \mathcal{M}_{\sigma}^{1}$ the subset of ergodic probability measures with compact support.

Proof. First we show

$$
\mathcal{P}_{\psi}\left(\varphi, \Sigma_{a}^{\mathrm{per}}\right) \geq \sup \left\{\frac{h_{\nu}(\sigma)}{\int \psi d \nu}+\frac{\int \varphi d \nu}{\int \psi d \nu}: \nu \in \mathcal{M}_{\sigma}^{1} \text { with } \varphi, \psi \in L^{1}(\nu)\right\} .
$$

By Corollary 2.8 we have $0 \geq \mathcal{P}_{1}\left(\varphi-\beta \psi, \Sigma_{a}^{\text {per }}\right)$ for $\beta>\mathcal{P}_{\psi}\left(\varphi, \Sigma_{a}^{\text {per }}\right)$. Using results from [Sar99, Theorem 3] for the Gurevič pressure, which turn out to be valid also for irreducible incidence matrices, we obtain

$$
\begin{aligned}
0 & \geq \mathcal{P}_{1}\left(\varphi-\beta \psi, \Sigma_{a}^{\mathrm{per}}\right) \\
& \geq \sup \left\{h_{\nu}(\sigma)+\int \varphi d \nu-\beta \int \psi d \nu: \nu \in \mathcal{M}_{\sigma}^{1} \text { with } \varphi, \psi \in L^{1}(\nu)\right\} \\
& =\sup \left\{\int \psi d \nu\left(\frac{h_{\nu}(\sigma)}{\int \psi d \nu}+\frac{\int \varphi d \nu}{\int \psi d \nu}-\beta\right): \nu \in \mathcal{M}_{\sigma}^{1} \text { with } \varphi, \psi \in L^{1}(\nu)\right\},
\end{aligned}
$$

and hence, $\mathcal{P}_{\psi}\left(\varphi, \Sigma_{a}^{\text {per }}\right) \geq \sup \left\{\frac{h_{\nu}(\sigma)}{\int \psi d \nu}+\frac{\int \varphi d \nu}{\int \psi d \nu}: \nu \in \mathcal{M}_{\sigma}^{1}\right.$ with $\left.\varphi, \psi \in L^{1}(\nu)\right\}$.

Next, we are going to prove that $\mathcal{P}_{\psi}\left(\varphi, \Sigma_{a}^{\text {per }}\right) \leq \sup \left\{\frac{h_{\nu}(\sigma)}{\int \psi d \nu}+\frac{\int \varphi d \nu}{\int \psi d \nu}: \nu \in \mathcal{E}_{\sigma, c}^{1}\right\}$. Since by Corollary 3.19 , $\left(\psi, \varphi, \Sigma_{a}^{\mathrm{per}}\right)$ satisfies the exhausting principle we may restrict our attention to the finite alphabet case. More precisely, we find a sequence of compact $\sigma$-invariant subsets $K_{n} \subset \Sigma$, such that $\lim _{n \rightarrow \infty} \mathcal{P}_{\psi, K_{n}}\left(\varphi, \Sigma_{a}^{\text {per }}\right)=$ 
$\mathcal{P}_{\psi}\left(\varphi, \Sigma_{a}^{\mathrm{per}}\right)$. For the finite alphabet case we have by Remark 2.11 and by the classical variational principle (cf. [Wal82, DGS76])

$$
\begin{aligned}
0 & =\mathcal{P}_{1, K_{n}}\left(\varphi-\mathcal{P}_{\psi, K_{n}}\left(\varphi, \Sigma_{a}^{\mathrm{per}}\right) \psi, \Sigma_{a}^{\mathrm{per}}\right) \\
& =\sup \left\{h_{\mu}+\int \varphi d \mu-\mathcal{P}_{\psi, K_{n}}\left(\varphi, \Sigma_{a}^{\mathrm{per}}\right) \int \psi d \mu: \mu \in \mathcal{E}_{\sigma, c}^{1}, \mu\left(K_{n}\right)=1\right\} \\
& =\sup \left\{\frac{h_{\mu}+\int \varphi d \mu}{\int \psi d \mu}: \mu \in \mathcal{E}_{\sigma, c}^{1}, \mu\left(K_{n}\right)=1\right\}-\mathcal{P}_{\psi, K_{n}}\left(\varphi, \Sigma_{a}^{\mathrm{per}}\right) .
\end{aligned}
$$

The fact that $\sup \left\{\frac{h_{\nu}(\sigma)}{\int \psi d \nu}+\frac{\int \varphi d \nu}{\int \psi d \nu}: \nu \in \mathcal{M}_{\sigma}^{1}\right.$ with $\left.\varphi, \psi \in L^{1}(\nu)\right\}$ is greater or equal to $\sup \left\{\frac{h_{\nu}(\sigma)}{\int \psi d \nu}+\frac{\int \varphi d \nu}{\int \psi d \nu}: \nu \in \mathcal{E}_{\sigma, c}^{1}\right\}$ then finishes the proof.

\section{Proof of Theorem 1.2}

Let $\Phi=\left(\varphi_{t}\right)_{t \in \mathbb{R}_{>0}}$ be the special semi-flow over

$$
Y:=\left\{(\omega, t) \in \Sigma \times \mathbb{R}^{+}: 0 \leq t \leq \tau(\omega)\right\} / \sim
$$

with height function $\tau: \Sigma \rightarrow \mathbb{R}_{>0}$ defined by $\varphi_{t}(\omega, s)=(\omega, s+t)$.

As a key lemma for the proof of Theorem 1.2 we show that $\mathbf{P}(g \mid \Phi)$ satisfies a certain variational principle, where the supremum is taken over the set $\mathcal{E}_{\sigma}^{1}(\tau)$ of ergodic $\sigma$-invariant probability measures $\mu$ on the base space satisfying $\int \tau d \mu<\infty$. Note, that this Lemma generalises [Sav98, Lemma 1] to our situation.

Lemma 4.1. If $\tau: \Sigma \rightarrow \mathbb{R}_{>0}$ is Hölder continuous satisfying $\sum_{i=0}^{\infty} \tau \circ \sigma^{i}=\infty$ and $g: Y \rightarrow \mathbb{R}$ has the property that $\Delta_{g}: \Sigma \rightarrow \mathbb{R}$ is Hölder continuous then we have

$$
\mathbf{P}(g \mid \Phi)=\sup \left\{\frac{h(\sigma, \mu)}{\int \tau d \mu}+\frac{\int \Delta_{g} d \mu}{\int \tau d \mu}: \mu \in \mathcal{E}_{\sigma}^{1}(\tau) \text { with } \Delta_{g} \in L^{1}(\mu)\right\} .
$$

Proof. This proof follows mainly the proof of the analogue statement in [Sav98]. Let us denote the set of $\sigma$-invariant ergodic measures on $\Sigma$ satisfying $\int \tau \mathrm{d} \mu<\infty$ by $\mathcal{E}_{\sigma}(\tau)$. Note, that there is a one-to-one correspondence between the $\Phi$-invariant ergodic probability measures on $Y$ and the normalised product measures $\overline{\mu \times \lambda}:=$ $\left.(\mu \times \lambda)\right|_{Y} / \int \tau \mathrm{d} \mu$ with $\mu \in \mathcal{E}_{\sigma}(\tau)$ and $\lambda$ denoting the Lebesgue measures on the fibers. By definition of $\mathbf{P}(g \mid \Phi)$ and the fact that $\left.\int g d(\mu \times \lambda)\right|_{Y}=\int \Delta_{g} d \mu$ we have

$$
\mathbf{P}(g \mid \Phi)=\sup \left\{h\left(\varphi_{1}, \overline{\mu \times \lambda}\right)+\frac{\int \Delta_{g} d \mu}{\int \tau \mathrm{d} \mu}: \mu \in \mathcal{E}_{\sigma}(\tau) \text { such that } \Delta_{g} \in L^{1}(\mu)\right\} .
$$

Since $\tau>0$ and $\sum_{i=0}^{\infty} \tau \circ \sigma^{i}=\infty$ we have by a result of Hopf [Hop37 that all elements of $\mathcal{E}_{\sigma}(\tau)$ are conservative. If $\mu$ is $\sigma$-finite ergodic invariant and conservative (not necessarily a probability) then $h(\sigma, \mu)$ will subsequently be understood as in Kre67. We then notice that $h\left(\varphi_{1}, \overline{\mu \times \lambda}\right)$ is equal to $h(\sigma, \mu) / \int \tau d \mu$ for all $\mu \in$ $\mathcal{E}_{\sigma}(\tau)$. This follows e.g. from [Sav98, Theorem 1] applied to the natural extension of $\left(Y, \varphi_{1}\right)$ and using the results on the relative metric entropy by Ledrappier and Walters [LW77]. We conclude that

$$
\mathbf{P}(g \mid \Phi)=\sup \left\{\frac{h(\sigma, \mu)}{\int \tau d \mu}+\frac{\int \Delta_{g} d \mu}{\int \tau \mathrm{d} \mu}: \mu \in \mathcal{E}_{\sigma}(\tau) \text { such that } \Delta_{g} \in L^{1}(\mu)\right\} .
$$


Let us now introduce the induced transformation on $E$ by setting

$$
\sigma_{E}^{*}(x):=\sigma^{N(x)}(x), x \in E,
$$

with $N(x):=\inf \left\{n \geq 1: \sigma^{n}(x) \in E\right\}$ denoting the first return time to the set $E$. As shown in [Kre67, 3.1], every Borel set $E$ with $0<\mu(E)<\infty$ is a sweep-out set (i.e. $\left.\mu\left(\Sigma \backslash \bigcup_{n \in \mathbb{N}} \sigma^{-n}(E)\right)=0\right)$ and hence we have $h(\sigma, \mu)=h\left(\sigma_{E}^{*}, \mu_{\mid E}\right)$.

Let us first assume that $\mathbf{P}(g \mid \Phi)<\infty$. Then for $\epsilon>0$ we find $\mu \in \mathcal{E}_{\sigma}(\tau)$ such that

$$
\mathbf{P}(g \mid \Phi)-\epsilon \leq \frac{h(\sigma, \mu)}{\int \tau d \mu}+\frac{\int \Delta_{g} d \mu}{\int \tau \mathrm{d} \mu} \leq \mathbf{P}(g \mid \Phi)<\infty .
$$

Since $\int \tau d \mu<\infty$ and $\tau>0$ we find $a \in \Sigma^{*}$ such that $[a]$ hase finite and positive measure with respect to $\mu$. We may normalise $\mu$ such that $\mu_{\mid[a]}=1$ without changing $\frac{h(\sigma, \mu)}{\int \tau d \mu}+\frac{\int \Delta_{g} d \mu}{\int \tau \mathrm{d} \mu}$. In this situation we then have $h(\sigma, \mu)=h\left(\sigma_{[a]}^{*}, \mu_{\mid[a]}\right)$.

Our aim is to construct a sequence of finite invariant ergodic measures $\left(\mu_{n, k}\right)_{n, k \in \mathbb{N}}$ on $\Sigma$, such that for $n, k$ large we have $h\left(\sigma, \mu_{n, k}\right) \geq h(\sigma, \mu)-\epsilon$ as well as

$$
\left|\int \Delta_{g} d \mu_{n, k}-\int \Delta_{g} \mathrm{~d} \mu\right|<\epsilon \text { and }\left|\int \tau d \mu_{n, k}-\int \tau \mathrm{d} \mu\right|<\epsilon,
$$

which then would finish the proof.

For this we define the countable set of words

$$
C^{*}:=\left\{\omega \in \Sigma^{*}: \omega a \in \Sigma^{*},[\omega a] \subset[a],\left.N\right|_{[\omega a]}=|\omega|\right\}=\left\{\gamma_{i}: i \in \mathbb{N}\right\}
$$

and let $C_{n}^{*}:=\left\{\gamma_{i} \in C^{*}: i \leq n\right\}$ denote the set of its first $n \in \mathbb{N}$ elements. We have that $\left([a], \sigma_{[a]}^{*}\right)$ is conjugated to the fullshift over the alphabet $C^{*}$, which we denote by $\left(\Sigma_{C^{*}}, \sigma^{*}\right)$. By the definition of the measure theoretical entropy we have for every $k \in \mathbb{N}$ and all $n \in \mathbb{N}$ sufficiently large

$$
h\left(\sigma_{[a]}^{*}, \mu_{\mid[a]}\right) \leq-\frac{1}{k} \sum_{\omega \in\left(C^{*}\right)^{k}} \mu([\omega]) \log \mu([\omega]) \leq-\frac{1}{k} \sum_{\omega \in\left(C_{n}^{*}\right)^{k}} \mu([\omega]) \log \mu([\omega])+\epsilon .
$$

Let $q_{n, k}:=\mu\left(\bigcup_{\omega \in\left(C_{n}^{*}\right)^{k}}[\omega]\right)$, which is finite for fixed $k \geq|a|$ and every $n \in \mathbb{N}$, and tends to 1 for $n \rightarrow \infty$. For $k \geq|a|$ and $n \in \mathbb{N}$ we define the Bernoulli measures $\mu_{n, k}^{*}$ on $\Sigma_{C^{*}}$ by requiring that $\mu_{n, k}^{*}([\omega]):=\mu([\omega]) / q_{n, k}$ for $\omega \in\left(C_{n}^{*}\right)^{k}$, and zero else. Its push forward to $\limsup _{n} \sigma^{-n}([a])$ will also be denoted by $\mu_{n, k}^{*}$. Hence, we obtain for the measure theoretical entropy of the induced system

$$
\begin{aligned}
h\left(\sigma_{[a]}^{*}, \mu_{n, k}^{*}\right) & =-\frac{1}{k} \sum_{\omega \in\left(C_{n}^{*}\right)^{k}} \mu_{n, k}^{*}([\omega]) \log \mu_{n, k}^{*}([\omega]) \\
& =-\frac{1}{k} \sum_{\omega \in\left(C_{n}^{*}\right)^{k}} \mu([\omega]) \log \mu([\omega])-\log q_{n, k},
\end{aligned}
$$

which tends to $-\frac{1}{k} \sum_{\omega \in\left(C^{*}\right)^{k}} \mu([\omega]) \log \mu([\omega])$ for $n \rightarrow \infty$. Combining this with the above estimate we have for all $k, n \in \mathbb{N}$ large enough that

$$
h\left(\sigma_{[a]}^{*}, \mu_{n, k}^{*}\right) \geq h\left(\sigma_{[a]}^{*}, \mu_{\mid[a]}\right)-\epsilon .
$$


We define for $n, k \in \mathbb{N}$ the $\sigma$-invariant and ergodic measure $\mu_{n, k}$ on $(\Sigma, \mathcal{B})$ by

$$
\mu_{n, k}(E):=\int \sum_{i=0}^{N(x)-1} \mathbb{1}_{E} \circ \sigma^{i}(x) \mathrm{d} \mu_{n, k}^{*}(x), E \in \mathcal{B}(\Sigma),
$$

which by Kac's formula Kac47 has finite total mass

$$
\mu_{n, k}(\Sigma)=\int N \mathrm{~d} \mu_{n, k}^{*}=\sum_{\omega \in\left(C_{n}^{*}\right)^{k}}\left|\omega_{1}\right| \mu([\omega])<\infty .
$$

The above estimates imply that for $k$ and $n$ sufficiently large

$$
h\left(\sigma, \mu_{n, k}\right)=h\left(\sigma_{[a]}^{*}, \mu_{n, k}^{*}\right) \geq h\left(\sigma_{[a]}^{*}, \mu_{\mid[a]}\right)-\epsilon=h(\sigma, \mu)-\epsilon .
$$

We also introduce the induced potentials $\tau^{*}, \Delta_{g}^{*}$ defined for $x \in \lim \sup _{n} \sigma^{-n}([a])$ by

$$
\tau^{*}(x):=\sum_{i=0}^{N(x)-1} \tau\left(\sigma^{i}(x)\right) \text { and } \Delta_{g}^{*}(x):=\sum_{i=0}^{N(x)-1} \Delta_{g}\left(\sigma^{i}(x)\right) .
$$

Recall that $\tau^{*}$ and $\Delta_{g}^{*}$ are defined $\mu$-a.e. on $[a]$. Since $\tau$ and $\Delta_{g}$ are Hölder continuous it follows by the remark at the beginning of Section 3.1 that $\tau^{*}$ and $\Delta_{g}^{*}$ are Hölder continuous as functions on $\left(C^{*}\right)^{\mathbb{N}}$. Hence we can choose $k$ sufficiently large, such that

$$
\sup \left\{\max \left\{\left|\tau^{*}(x)-\tau^{*}(y)\right|,\left|\Delta_{g}^{*}(x)-\Delta_{g}^{*}(y)\right|\right\}: x, y \in[\omega], \omega \in\left(C^{*}\right)^{k}\right\}<\epsilon / 2 .
$$

Consequently, we have

$$
\left|\int \tau^{*} \mathrm{~d} \mu_{n, k}^{*}-\int \tau^{*} \mathbb{1}_{\omega \in\left(C_{n}^{*}\right)^{k}}[\omega] \mathrm{d} \mu_{\mid[a]}\right|<\epsilon / 2
$$

and

$$
\left|\int \Delta_{g}^{*} \mathrm{~d} \mu_{n, k}^{*}-\int \Delta_{g}^{*} \mathbb{1}_{\omega \in\left(C_{n}^{*}\right)^{k}}[\omega] \mathrm{d} \mu_{\mid[a]}\right|<\epsilon / 2 .
$$

By Kac's formula we have $\int \tau \mathrm{d} \mu=\int \tau^{*} \mathrm{~d} \mu_{\mid[a]}$ and $\int \Delta_{g} \tau \mathrm{d} \mu=\int \Delta_{g}^{*} \mathrm{~d} \mu_{\mid[a]}$. The latter implies that $\tau^{*}$ and $\Delta_{g}^{*}$ are $\mu_{\mid[a]}$-integrable. Since $q_{n, k}$ tends to 1 for $n \rightarrow \infty$, we have for large $n$

$$
\left|\int \tau^{*} \mathbb{1}_{\bigcup_{\omega \in\left(C_{n}^{*}\right)^{k}}[\omega]} \mathrm{d} \mu_{\mid[a]}-\int \tau^{*} \mathrm{~d} \mu_{\mid[a]}\right|<\epsilon / 2
$$

and

$$
\left|\int \Delta_{g}^{*} \mathbb{1}_{\omega \in\left(C_{n}^{*}\right)^{k}}[\omega] \mathrm{d} \mu_{\mid[a]}-\int \Delta_{g}^{*} \mathrm{~d} \mu_{\mid[a]}\right|<\epsilon / 2 .
$$

Again by Kac's formula we have $\int \tau \mathrm{d} \mu_{n, k}=\int \tau^{*} \mathrm{~d} \mu_{n, k}^{*}$ and $\int \Delta_{g} \mathrm{~d} \mu_{n, k}=\int \Delta_{g}^{*} \mathrm{~d} \mu_{n, k}^{*}$. Combining this with the above estimates we obtain $\left|\int \tau \mathrm{d} \mu_{n, k}-\int \tau \mathrm{d} \mu\right|<\epsilon$ as well as $\left|\int \Delta_{g} \mathrm{~d} \mu_{n, k}-\int \Delta_{g} \mathrm{~d} \mu\right|<\epsilon$.

Now the proof for the case $\mathbf{P}(g \mid \Phi)=\infty$ follows along the same lines.

Proof of Theorem 1.2. By Lemma 4.1 we have

$$
\mathbf{P}(g \mid \Phi)=\sup \left\{\frac{h(\sigma, \mu)}{\int \tau d \mu}+\frac{\int \Delta_{g} d \mu}{\int \tau d \mu}: \mu \in \mathcal{E}_{\sigma}^{1}(\tau) \text { with } \Delta_{g} \in L^{1}(\mu)\right\} .
$$


The variational principle for the $\psi$-induced pressure obtained in Proposition 3.26 applied to the irreducible components of $\Sigma$ then shows that the latter is equal to $\sup _{a \in I} \mathcal{P}_{\tau}\left(\Delta_{g}, \Sigma_{a}^{\text {per }}\right)$. The second equality follows from Corollary $3.23(2)$.

Remark 4.2. We would finally like to remark that if $\tau$ is bounded away from zero, then there is a one-to-one correspondents between the $\Phi$-invariant probability measures on $Y$ and the set $\mathcal{M}_{\sigma}^{1}$. Using the first equality in Proposition 3.26 it follows that the value of $\mathbf{P}(g \mid \Phi)$ stays unchanged if we replace $\mathcal{E}_{\Phi}^{1}$ by the larger set $\mathcal{M}_{\Phi}^{1}$.

\section{REFERENCES}

[BI06] L. Barreira and G. Iommi, Suspension flows over countable Markov shifts, J. Stat. Phys. 124 (2006), no. 1, 207-230. MR MR2256622 (2008f:37070)

[Bro85] R. Brooks, The bottom of the spectrum of a Riemannian covering, J. Reine Angew. Math. 357 (1985), 101-114. MR 783536 (86h:58138)

[Coh82] J. M. Cohen, Cogrowth and amenability of discrete groups, J. Funct. Anal. 48 (1982), no. 3, 301-309. MR MR678175 (85e:43004)

[DGS76] M. Denker, Chr. Grillenberger, and K. Sigmund, Ergodic theory on compact spaces, Lecture Notes in Mathematics, Vol. 527, Springer-Verlag, Berlin, 1976. MR MR0457675 (56 \#15879)

[DL09a] D.A. Dastjerdi and S. Lamei, Generating function for special flows over the 1-step countable topological Markov chains, preprint (2009).

[DL09b] _ Observing geometric codes from phase space, preprint (2009).

[GdlH01] R. Grigorchuk and P. de la Harpe, Limit behaviour of exponential growth rates for finitely generated groups, Essays on geometry and related topics, Vol. 1, 2, Monogr. Enseign. Math., vol. 38, Enseignement Math., Geneva, 2001, pp. 351-370. MR MR1929333 (2003h:20078)

[GK01] B. M. Gurevič and S. Katok, Arithmetic coding and entropy for the positive geodesic flow on the modular surface, Mosc. Math. J. 1 (2001), no. 4, $569-582,645$, Dedicated to the memory of I. G. Petrovskii on the occasion of his 100th anniversary. MR MR1901076 (2003h:37040)

[Gri80] R. Grigorchuk, Symmetrical random walks on discrete groups, Multicomponent random systems, Adv. Probab. Related Topics, vol. 6, Dekker, New York, 1980, pp. 285-325. MR MR599539 (83k:60016)

[Gur69] B. M. Gurevič, Topological entropy of a countable Markov chain, Dokl. Akad. Nauk SSSR 187 (1969), 715-718. MR MR0263162 (41 \#7767)

[Gur70] _ Shift entropy and Markov measures in the space of paths of a countable graph, Dokl. Akad. Nauk SSSR 192 (1970), 963-965. MR MR0268356 (42 \#3254)

[Hop37] E. Hopf, Ergodentheorie, Ergebnisse der Mathematik und ihrer Grenzgebiete 5, H. 2, J. Springer, Berlin, 1937.

[HU99] P. Hanus and M. Urbański, A new class of positive recurrent functions, Geometry and topology in dynamics (Winston-Salem, NC, 1998/San Antonio, TX, 1999), Contemp. Math., vol. 246, Amer. Math. Soc., Providence, RI, 1999, pp. 123-135. MR MR1732376 (2000j:37006)

[Iom10] G. Iommi, Thermodynamic formalism for the positive geodesic flow on the modular surface, preprint (2010). 
[JK11] J. Jaerisch and M. Kesseböhmer, Regularity of multifractal spectra of conformal iterated function systems, Trans. Amer. Math. Soc. 363 (2011), no. 1, 313-330.

[Kac47] M. Kac, On the notion of recurrence in discrete stochastic processes, Bull. Amer. Math. Soc. 53 (1947), 1002-1010. MR 0022323 (9,194a)

[Kes59a] H. Kesten, Full Banach mean values on countable groups, Math. Scand. 7 (1959), 146-156. MR MR0112053 (22 \#2911)

[Kes59b] _ Symmetric random walks on groups, Trans. Amer. Math. Soc. 92 (1959), 336-354. MR MR0109367 (22 \#253)

[Kes67] _ , The Martin boundary of recurrent random walks on countable groups, Proc. Fifth Berkeley Sympos. Math. Statist. and Probability (Berkeley, Calif., 1965/66), Univ. California Press, Berkeley, Calif., 1967, pp. Vol. II: Contributions to Probability Theory, Part 2, pp. 51-74. MR MR0214137 (35 \#4988)

[Kes01] M. Kesseböhmer, Large deviation for weak Gibbs measures and multifractal spectra, Nonlinearity 14 (2001), no. 2, 395-409. MR MR1819804 (2002a:60037)

[KMS10] M. Kesseböhmer, S. Munday, and B. O. Stratmann, Strong renewal theorems and Lyapunov spectra for $\alpha$-Farey and $\alpha$-Lüroth systems, arXiv (2010).

[Kre67] U. Krengel, Entropy of conservative transformations, Z. Wahrscheinlichkeitstheorie und Verw. Gebiete 7 (1967), 161-181. MR 0218522 (36 \#1608)

[KS07] M. Kesseböhmer and B. O. Stratmann, Homology at infinity; fractal geometry of limiting symbols for modular subgroups, Topology 46 (2007), no. 5, 469-491. MR MR2337557 (2009a:37096)

[LW77] F. Ledrappier and P. Walters, A relativised variational principle for continuous transformations, J. London Math. Soc. (2) 16 (1977), no. 3, 568576. MR 0476995 (57 \#16540)

[MU01] R. D. Mauldin and M. Urbański, Gibbs states on the symbolic space over an infinite alphabet, Israel J. Math. 125 (2001), 93-130. MR MR1853808 (2002k:37048)

[MU03] _ Graph directed Markov systems, Cambridge Tracts in Mathematics, vol. 148, Cambridge University Press, Cambridge, 2003, Geometry and dynamics of limit sets. MR MR2003772 (2006e:37036)

[Prz99] F. Przytycki, Conical limit set and Poincaré exponent for iterations of rational functions, Trans. Amer. Math. Soc. 351 (1999), no. 5, 2081-2099. MR MR1615954 (99h:58110)

[RSU08] M. Roy, H. Sumi, and M. Urbański, Analytic families of holomorphic iterated function systems, Nonlinearity 21 (2008), no. 10, 2255-2279. MR MR2439479 (2009h:37096)

[Sar99] O. M. Sarig, Thermodynamic formalism for countable Markov shifts, Ergodic Theory Dynam. Systems 19 (1999), no. 6, 1565-1593. MR MR1738951 (2000m:37009)

[Sar01] _ Thermodynamic formalism for null recurrent potentials, Israel J. Math. 121 (2001), 285-311. MR MR1818392 (2001m:37059)

[Sar03] _ Existence of Gibbs measures for countable Markov shifts, Proc. Amer. Math. Soc. 131 (2003), no. 6, 1751-1758 (electronic). 
MR MR1955261 (2004b:37056)

[Sav98] S. V. Savchenko, Special flows constructed from countable topological Markov chains, Funktsional. Anal. i Prilozhen. 32 (1998), no. 1, 40-53, 96. MR MR1627271 (99m:28040)

[Wal82] P. Walters, An introduction to ergodic theory, Graduate Texts in Mathematics, vol. 79, Springer-Verlag, New York, 1982. MR MR648108 (84e:28017)

E-mail address: jogy@math.uni-bremen.de

E-mail address: mhk@math.uni-bremen.de

E-mail address: lamei@guilan.ac.ir

Fachbereich Mathematik, Universität Bremen, 28359 Bremen, Germany

Department of Mathematics, The University of Guilan, P.O. Box 1841, Rasht, Iran. 